\title{
GÉNÉRICITÉ D’EXPOSANTS DE LYAPUNOV NON-NULS POUR DES PRODUITS DÉTERMINISTES DE MATRICES
}

\section{GENERICITY OF NON-ZERO LYAPUNOV EXPONENTS FOR DETERMINISTIC PRODUCTS OF MATRICES}

\author{
Christian BONATTI $^{\mathrm{a}}$, Xavier GÓMEZ-MONT ${ }^{\mathrm{b}}$, Marcelo VIANA $^{\mathrm{c}, *, 1}$ \\ ${ }^{a}$ Laboratoire de topologie, UMR 5584 du CNRS, B.P. 47 870, 21078 Dijon Cedex, France \\ ${ }^{\mathrm{b}}$ CIMAT, C.P. 36240, Guanajuato, Gto.; Mexico \\ ${ }^{\mathrm{c}}$ IMPA, Est. D. Castorina 110, 22460-320 Rio de Janeiro, Brazil
}

Reçu en 5 novembre 2001

ABSTRACT. - We propose a geometric sufficient criterium “à la Furstenberg” for the existence of non-zero Lyapunov exponents for certain linear cocycles over hyperbolic transformations: non-existence of probability measures on the fibers invariant under the cocycle and under the holonomies of the stable and unstable foliations of the transformation. This criterium applies to locally constant and to dominated cocycles over hyperbolic sets endowed with an equilibrium state.

As a consequence, we get that non-zero exponents exist for an open dense subset of these cocycles, which is also of full Lebesgue measure in parameter space for generic parametrized families of cocycles.

This criterium extends to continuous time cocycles obtained by lifting a hyperbolic flow to a projective fiber bundle, tangent to some foliation transverse to the fibers. Again, non-zero Lyapunov exponents are implied by non-existence of transverse measures invariant under the holonomy of the foliation.

We apply this last result to a natural geometric context: the geodesic flow tangent to the leaves of a foliation obtained as the suspension of a representation $\rho: \pi_{1}(S) \rightarrow \operatorname{PSL}(2, \mathbb{C})$ of the fundamental group of a hyperbolic compact surface. We prove the existence of non-zero Lyapunov exponents for the corresponding cocycle, for $\rho$ in a dense open subset of all the representations. As a consequence we get that this foliated geodesic flow has a unique SinaiRuelle-Bowen measure.

\footnotetext{
* Corresponding author.

E-mail addresses: bonatti@u-bourgogne.fr (C. Bonatti), gmont@ cimat.mx (X. Gómez-Mont), viana@impa.br (M. Viana).

${ }^{1}$ Avec le soutien financier de CONACYT 28541-E, CIMAT, CNRS, Université de Bourgogne, CNPq001/2000, Faperj, PRONEX-Sistemas Dinâmicos, IMPA.
} 
(c) 2003 L'Association Publications de l'Institut Henri Poincaré. Published by Elsevier B.V. All rights reserved

MSC: 37H15; 37D30; 37D40

Keywords: Linear cocycle; Lyapunov exponents; Vector bundle; Projective bundle; Holomorphic foliation; Group representations

RÉSUMÉ. - Pour garantir l'existence d'exposants de Lyapunov non-nuls pour certains cocycles linéaires au-dessus de dynamiques hyperboliques, nous proposons un critère géométrique "à la Furstenberg" : la non-existence d'une famille de probabilités dans les fibres, invariante par l'action du cocycle et par les holonomies de feuilletages stable et instable. Ce critère s'applique quand le cocycle est localement constant ou dominé, et quand la base est un ensemble hyperbolique muni d'un état d'équilibre.

En conséquence nous montrons l'existence d'exposants non-nuls pour un ouvert dense de l'ensemble de ces cocycles, qui a mesure de Lebesgue totale dans l'espace des paramètres pour une famille générique de tels cocycles.

Ce critère s'adapte aux cocycles en temps continu obtenus en relevant un flot hyperbolique sur les feuilles d'un feuilletage transversalement projectif, transverse aux fibres d'un fibré projectif. De nouveau, l'existence d'exposants non-nuls est impliquée par la non-existence d'une mesure transverse invariante par les holonomies du feuilletage.

Nous appliquons ce dernier résultat au contexte géométrique suivant : le flot géodésique tangent aux feuilles d'un feuilletage obtenu par suspension d'une représentation $\rho: \pi_{1}(S) \rightarrow P S L(2, \mathbb{C})$ du groupe fondamental d'une surface compacte hyperbolique $S$. Nous montrons l'existence d'exposants non-nuls pour le cocycle correspondant, pour un ouvert dense de toutes les représentations $\rho$. En conséquence nous obtenons que le flot géodésique feuilleté possède une unique mesure de Sinaï-Ruelle-Bowen.

(C) 2003 L'Association Publications de l'Institut Henri Poincaré. Published by Elsevier B.V. All rights reserved

Mots Clés: Cocycle linéaire; Exposants de Lyapunov; Fibré vectoriel; Fibré projectif; Feuilletage holomorphe; Representation de groupes

\section{Introduction}

Ce travail a deux motivations très différentes, qui proviennent de deux projets a priori indépendants :

1. Feuilletages holomorphes: d'une part, on aimerait comprendre le comportement statistique des feuilles des feuilletages holomorphes, en commençant par les feuilletages les plus simples : les feuilletages définis comme la suspension d'une représentation d'holonomie à valeurs dans $S L(k, \mathbb{C})$.

2. Dynamiques partiellement hyperboliques : d'autre part, l'une des difficultés pour comprendre le comportement statistique d'une dynamique partiellement hyperbolique est le contrôle des exposants de Lyapunov dans la direction centrale. Plus précisément on aimerait montrer que, génériquement, ces exposants sont non-nuls.

Le lien entre ces deux sujets provient de ce que, pour les feuilletages que nous avons considérés, le flot géodésique tangent au feuilletage est hyperbolique le long des feuilles, la direction transverse faisant office de "direction centrale", et les exposants de Lyapunov dans cette direction centrale permettront de caractériser le comportement asymptotique des feuilles du feuilletage. 
Dans ces deux contextes, le comportement dans la direction centrale est donné par un produit d'applications linéaires, ce produit n'étant pas aléatoire indépendant puisque donné par une dynamique déterministe, mais gardant cependant un certain degré d'indépendance, due au caractère chaotique de la dynamique de la base.

Pour les produits aléatoires et indépendants de matrices de $S L(k, \mathbb{C})$, un célèbre travail de Furstenberg (voir [10]) montre que, ou bien l'action de ces matrices sur l'espace projectif laisse invariante une même probabilité (ce qui est une condition très forte), ou bien pour presque tout tirage la norme du produit croit exponentiellement : en d'autres termes, presque tout tirage possède un exposant de Lyapunov non-nul. Ce travail complétait de façon très satisfaisante le résultat fondamental d'Oseledets [20] qui assurait que les exposants de Lyapunov étaient bien définis.

Depuis, de nombreux auteurs ont proposé des généralisations du travail de Furstenberg. Par exemple, Guivarc'h et Raugi [11] montrent que, génériquement, chaque exposant de Lyapunov est de multiplicité 1 . Dans $[23,18,17]$, Royer et Ledrappier affaiblissent l'hypothèse d'indépendance du tirage des matrices.

On pourrait espérer que ces résultats se généralisent au cas des cocycles à valeurs dans $S L(k, \mathbb{C})$ au-dessus d'une dynamique hyperbolique, c'est-à-dire que l'on multiplie des matrices dont le tirage est donné par une dynamique hyperbolique. Cependant, un théorème récent de Bochi [3] montre que l'existence d'exposants de Lyapunov nuls est générique pour les cocycles continus à valeurs dans $S L(2, \mathbb{C})$, non uniformément hyperboliques, au-dessus d'une dynamique $f$ munie d'une mesure ergodique nonatomique $\mu$ quelconque. En fait, il obtient ce résultat aussi dans le cadre, beaucoup plus délicat, du cocycle donné par la différentielle d'un difféomorphisme de classe $C^{1}$, préservant l'aire, sur une surface.

Ces théorèmes de Bochi montrent que les résultats sur les produits aléatoires ne peuvent pas s'adapter au cas de produits déterministes sans hypothèses supplémentaires. Nos conditions portent, d'une part sur la dynamique déterministe qui dirige le cocycle, que nous supposerons uniformément hyperbolique, et d'autre part sur la régularité du cocycle : il pourra être localement constant, ou bien Hölder continu et dominé. Cette dernière propriété veut dire que la dilatation et la contraction dans les fibres sont strictement moins fortes que celles de la dynamique de la base.

Les versions complètes de nos résultats sur les cocycles apparaîtront dans les sections qui suivent. Ici nous présentons leurs conséquences géométriques sur les deux sujets qui nous avaient motivés.

\subsection{Dynamiques partiellement hyperboliques}

Pour des dynamiques partiellement hyperboliques dont l'action dans la direction centrale définit un cocycle, nous montrons que les exposants de ce cocycle sont génériquement non-nuls. Avant d'énoncer nos résultats de façon précise, rappelons les notions d'hyperbolicité uniforme et partielle, de cocycle linéaire et projectif, et d'exposants de Lyapunov.

Soit $f$ un difféomorphisme d'une variété $M$. Étant donné un ensemble compact $f$ invariant $K$, on dit qu'une décomposition $T_{K} M=E^{1} \oplus E^{2}$ de l'espace tangent au-dessus 
de $K$ en deux sous-fibrés continus et $D f$-invariants est dominée s'il existe $N \geqslant 1$ tel que

$$
\frac{\left\|D f^{N}(x) v_{2}\right\|}{\left\|v_{2}\right\|} \leqslant \frac{1}{2} \frac{\left\|D f^{N}(x) v_{1}\right\|}{\left\|v_{1}\right\|}
$$

pour tout $x \in K$ et tous vecteurs non-nuls $v_{1} \in E_{x}^{1}$ et $v_{2} \in E_{x}^{2}$.

On dit que $K$ est uniformément hyperbolique s'il admet une décomposition dominée de l'espace tangent telle que $E^{1}$ soit uniformément dilatant et $E^{2}$ soit uniformément contractant. C'est à dire que, quitte à augmenter $N$, on a

$$
\left\|D f^{-N}(x) v_{1}\right\| \leqslant \frac{1}{2}\left\|v_{1}\right\| \quad \text { et } \quad\left\|D f^{N}(x) v_{2}\right\| \leqslant \frac{1}{2}\left\|v_{2}\right\|
$$

pour tout $v_{1} \in E_{x}^{1}, v_{2} \in E_{x}^{2}$ et $x \in K$.

Plus généralement, on dit que $K$ est partiellement hyperbolique s'il admet une décomposition dominée de l'espace tangent telle que $E^{1}$ soit uniformément dilatant, ou que $E^{2}$ soit uniformément contractant. Dans le premier cas on note $E^{1}=E^{u u}$ et, d'après la théorie de l'hyperbolicité normale (voir $[9,13]$ ), il existe alors un unique feuilletage $f$-invariant $\mathcal{F}^{u u}$ tangent à $E^{u u}$ en tout point de $K$, et appelé feuilletage instable-fort. Dans le deuxième cas on parle du feuilletage stable-fort $\mathcal{F}^{s s}$.

On dit que $K$ est fortement partiellement hyperbolique s'il admet une décomposition $D f$-invariante en trois sous-fibrés continus $T_{K}=E^{u u} \oplus E^{c} \oplus E^{s s}$ telle que $E^{u u}$ est uniformément dilatant, $E^{s s}$ est uniformément contractant, et les deux décompositions

$$
\left(E^{u u} \oplus E^{c}\right) \oplus E^{s s} \quad \text { et } \quad E^{u u} \oplus\left(E^{c} \oplus E^{s s}\right)
$$

sont dominées. On appelle $E^{c}$ la direction centrale.

Un ensemble basique hyperbolique $K$ est un ensemble uniformément hyperbolique transitif (existence d'orbites denses) et qui est l'ensemble maximal invariant dans un voisinage. On munit la restriction de $f$ à $K$ de la classe des états d'équilibre associés à des potentiels Hölder continus (voir [7] pour une définition précise et les propriétés des états d'équilibre). Ce sont des mesures de probabilité $f$-invariantes et ergodiques, dont le support coïncide avec $K$.

Étant donné un homéomorphisme $f: M \rightarrow M$ d'un espace topologique $M$, on appelle cocycle (continu) linéaire (respectivement projectif) au-dessus de $f$, la donnée de :

1. un fibré continu localement trivial $\pi: \mathcal{E} \rightarrow M$ de fibre $\mathbb{C}^{k}$ (respectivement $\left.\mathbb{C P}^{k-1}\right), k \geqslant 2$

2. un homéomorphisme $F: \mathcal{E} \rightarrow \mathcal{E}$ tel que $\pi \circ F=f \circ \pi$ et dont la restriction $F_{x}$ à toute fibre $\mathcal{E}_{x}=\pi^{-1}(x), x \in M$ est un isomorphisme linéaire (respectivement projectif) $\operatorname{sur} \mathcal{E}_{f(x)}$.

Les itérés $F^{n}$ d'un cocycle $F: \mathcal{E} \rightarrow \mathcal{E}$ au-dessus d'un homéomorphisme $f$ sont des cocycles au-dessus des $f^{n}$ définis par

$$
F_{x}^{n}=F_{f^{n-1}(x)} \cdots F_{x} \quad \text { et } \quad F_{x}^{-n}=F_{f^{-n}(x)}^{-1} \cdots F_{f^{-1}(x)}^{-1}
$$

pour chaque $n \in \mathbb{N}$. 
Si $f$ laisse invariante une mesure de probabilité $\mu$ telle que les deux fonctions $x \mapsto \log \left\|F_{x}\right\|$ et $x \mapsto \log \left\|F_{x}^{-1}\right\|$ sont $\mu$-intégrables, alors le Théorème d'Oseledets [20] affirme que, pour $\mu$-presque tout point $x$ dans $M$, il existe une décomposition de la fibre $\mathcal{E}_{x}=E_{x}^{1} \oplus \cdots \oplus E_{x}^{l(x)}$ en des sous-espaces linéaires, et il existe des nombres réels $\lambda_{1}(x), \ldots, \lambda_{l(x)}(x)$ tels que

$$
\lim _{n \rightarrow \pm \infty} \frac{1}{n} \log \left\|F_{x}^{n} v\right\|=\lambda_{j}(x)
$$

pour tout vecteur non-nul $v \in E_{x}^{j}$. On appelle les $\lambda_{j}(x)$ les exposants de Lyapunov du cocycle au point $x$. De plus, si la mesure $\mu$ est ergodique les exposants de Lyapunov, et leur nombre $l$, sont constants sur un ensemble de $\mu$-mesure totale : on parle alors d'exposants de Lyapunov du cocycle pour la mesure $\mu$.

A priori la notion d'exposants de Lyapunov pour un cocycle linéaire dépend du choix de métriques dans les fibres. Cependant, si $M$ est un espace compact et si l'on se restreint à des choix continus de métriques on se convainc facilement que les exposants de Lyapunov ne dépendent pas du choix de la métrique. On peut donc parler sans ambigüité des exposants de Lyapunov d'un cocycle linéaire à un point ou pour une mesure ergodique. On supposera désormais que $M$ est compacte. Remarquons que dans ce cadre la condition d'intégrabilité dans le Théorème d'Oseledets est automatiquement satisfaite (pour les cocycles continus).

Si $\pi: \mathcal{E} \rightarrow M$ est un fibré projectif obtenu par projectivisation d'un fibré linéaire alors, par définition, les exposants de Lyapunov d'un cocycle projectif $F: \mathcal{F} \rightarrow \mathcal{F}$ sont les exposants de Lyapunov d'un cocycle linéaire à valeurs dans $S L(k, \mathbb{C})$ dont $F$ soit le projectivisé : comme la projection naturelle $S L(k, \mathbb{C}) \rightarrow \operatorname{PSL}(k, \mathbb{C})$ a son noyau engendré par l'homothétie de rapport $\mathrm{e}^{2 i \pi / k}$, qui est une isométrie, la définition ne dépend pas du choix du cocycle linéaire.

Un fibré projectif $\pi: \mathcal{E} \rightarrow M$ arbitraire n'est pas a priori le projectivisé d'un fibré linéaire : de nouveau, l'ambigüité vit dans le groupe cyclique engendré par l'homothétie de rapport $\mathrm{e}^{2 i \pi / k}$. Voici en quelques mots comment définir les exposants de Lyapunov pour un cocycle projectif en général. On considère une partition de $M$ par un nombre fini d'ensembles $M_{i}$ d'adhérence incluse dans des ouverts $U_{i}$ trivialisant la fibration $\mathcal{E}$. Au-dessus de ces ensembles le fibré est trivial, donc est le projectivisé du fibré linéaire trivial, que l'on munit de la norme standard. Dans ces cartes $M_{i}$ on peut écrire le cocycle projectif comme le projectivisé d'un cocycle engendré par une application $A: M_{i} \rightarrow S L(k, \mathbb{C})$. On vérifie sans peine que les exposants de Lyapunov ne dépendent pas des choix des cartes trivialisantes (l'important étant que les ensembles $M_{i}$ soient relativement compacts dans les ouverts trivialisants $U_{i}$ ).

THÉORÈME 1. - Soit $f: M \rightarrow M$ un difféomorphisme de classe $C^{1}$ et $\Lambda$ un ensemble basique hyperbolique de $f$. Soit $\pi: \mathcal{E} \rightarrow M$ un fibré projectif lisse (de classe $C^{1}$ ) de fibre $\mathbb{C P}^{k-1}$ et $F: \mathcal{E} \rightarrow \mathcal{E}$ un cocycle projectif de classe $C^{1}$ au-dessus de $f$.

On suppose que $\pi^{-1}(\Lambda)$ est fortement partiellement hyperbolique de direction centrale tangente aux fibres (on parle de cocycle dominé au-dessus de $\Lambda$ ). Alors :

1. ou bien le cocycle projectif $F$ possède des exposants de Lyapunov non-nuls pour tout état d'équilibre $\mu$ associé à un potentiel Hölder continu sur $\Lambda$, 
2. ou bien il existe une famille continue $\left(m_{x}\right)_{x \in \Lambda}$ de probabilités dans les fibres $\mathcal{E}_{x}$ qui est invariante par l'action de $F$ et par les holonomies des feuilletages instable et stable-forts.

La seconde possibilité dans la conclusion du Théorème 1 est très rigide et instable. Cela nous permet de montrer :

THÉORÈME 2.- Dans l'espace des applications F vérifiant les hypothèses du Théorème 1, il existe un sous-ensemble ouvert dense pour la topologie $C^{1}$ pour lesquels le cocycle possède des exposants de Lyapunov non-nuls pour tout état d'équilibre $\mu$ d'un potentiel hölderien pour $(f, \Lambda)$.

Dans le Théorème 2, la conclusion reste vraie dans l'espace des application $F$ de classe $C^{r}$, avec $r \in[1,+\infty]$, muni de la topologie $C^{r}$. D'autre part on peut affaiblir l'hypothèse de régularité en supposant que $F$ est seulement Hölder continue. Dans ce cas on considère la topologie $C^{0}$ dans l'espace des applications Hölder continues avec les deux constantes d'Hölder fixées (voir la Remarque 2.11).

La conclusion reste vraie aussi dans le cadre des cocycles réels, c'est à dire, quand on prend des fibrés $\mathcal{E}$ de fibre $\mathbb{R} \mathbb{P}^{k-1}$, et qu'on suppose que l'action de $F$ dans les fibres est projective réel (voir le Corollaire 1.19 et la Remarque 2.12).

De plus, nous montrons que les cocycles dont les exposants de Lyapunov sont nuls, pour au moins un état d'équilibre, sont non typiques aussi d'un point de vue probabiliste : ils correspondent à des ensembles de mesure de Lebesgue nulle dans l'espace des paramètres, pour des familles paramétrées génériques (nombre fini de paramètres) :

Proposition 0.1. - Il existe un ouvert dense de familles paramétrées $t \mapsto F_{t}$ de cocycles projectifs dominés de classe $C^{r}, r \geqslant 1$, au dessus de $(f, \Lambda)$ pour lesquelles l'ensemble des paramètres $t$ tels que les exposants de Lyapunov de $F_{t}$ sont nuls (pour un état d'équilibre d'un potentiel hölderien) est discret et donc de mesure de Lebesgue nulle.

\subsection{Dynamiques tangentes à un feuilletage}

Étant donnée une variété différentiable $V$ munie d'un flot $X=\left\{X_{t}\right\}_{t \in \mathbb{R}}$, on appelle cocycle projectif au-dessus de $X$, la donnée de :

1. un fibré projectif $\pi: \mathcal{E} \rightarrow V$ de fibre $\mathbb{C P}^{k-1}, k \geqslant 2$;

2. un flot $Y=\left\{Y_{t}\right\}_{t \in \mathbb{R}}$ sur $\mathcal{E}$ tel que, pour tout $t \in \mathbb{R}$, l'application $Y_{t}$ est un cocycle projectif au-dessus de $X_{t}$.

Les exposants de Lyapunov d'un point $x \in V$, ou d'une mesure invariante ergodique $\mu$, du flot $X$ pour le cocycle $Y$ au-dessus de $X$ sont définis de façon identique aux exposants de Lyapunov d'un cocycle projectif au-dessus d'une transformation $f$; de plus ils sont obtenus en multipliant par $\frac{1}{t}$ ceux de $x$ ou de $\mu$ pour le cocycle $Y_{t}$ au-dessus de $X_{t}$ (ce produit ne dépendant pas de $t \neq 0$ ).

THÉORÈME 3. - Soit $V$ une variété compacte munie d'un flot d'Anosov transitif $X$ et soit $\pi: \mathcal{E} \rightarrow V$ un fibré projectif de fibre $\mathbb{C} \mathbb{P}^{k-1}$ admettant un feuilletage $\mathcal{F}$ transverse aux fibres et transversalement projectif. Notons $Y$ le relevé de $X$ sur les feuilles de $\mathcal{F}$. Alors l'une des deux propriétés suivantes est vérifiée : 
- Le feuilletage $\mathcal{F}$ admet une mesure transverse invariante par holonomie.

- Pour tout état d'équilibre $\mu$ de $X$ associé à un potentiel höldérien, le cocycle projectif induit par $Y$ au-dessus de $X$ possède un exposant de Lyapunov non-nul.

Dans l'énoncé ci-dessus le flot de $Y$ préserve les fibres et son action dans les fibres est donnée par les holonomies de $\mathcal{F}$ le long des orbites de $X$, donc elle est projective. C'est pourquoi on peut parler du cocycle projectif induit par $Y$ au-dessus de $X$.

Ici nous nous intéressons spécialement au cas des feuilletages obtenus comme suspension d'une représentation $\rho: \pi_{1}(S) \rightarrow \operatorname{PSL}(k, \mathbb{C})$ (morphisme contravariant) du groupe fondamental d'une surface de Riemann compacte. C'est un feuilletage $\mathcal{F}_{\rho}$ tranverse à une fibration $\pi_{\rho}: M_{\rho} \rightarrow S$ de fibre $\mathbb{C P}^{k-1}$ et dont l'holonomie au-dessus de chaque chemin $\gamma$ est donné par $\rho(\gamma)$; voir [16] pour une définition précise et les propriétés fondamentales de ces feuilletages. On note $X_{\rho}$ le flot géodésique tangent aux feuilles du feuilletage $\mathcal{F}_{\rho}$ : c'est le relevé sur les feuilles de $\mathcal{F}_{\rho}$ du flot géodésique $X$ de $S$.

ThÉORÈME 4. - Soit $\rho: \pi_{1}(S) \rightarrow P S L(k, \mathbb{C})$ une représentation du groupe fondamental d'une surface de Riemann compacte $S$ de genre $g \geqslant 2$. Alors l'une des propriétés suivantes est vérifiée:

- ou bien il existe une probabilité $\theta$ sur $\mathbb{C P}^{k-1}$ invariante par toutes les transformations $\rho(\gamma), \gamma \in \pi_{1}(S)$ : en d'autre termes, le feuilletage $\mathcal{F}_{\rho}$ possède une mesure transverse invariante par holonomie,

- ou bien le cocycle projectif défini par le flot géodésique feuilleté $X_{\rho}$ au-dessus de $X$ possède des exposants de Lyapunov non-nuls pour la mesure de Liouville.

Le fait que $S$ soit une surface hyperbolique n'est pas essentiel : le Théorème 4 reste vrai pour toute variété, de dimension quelconque, dont le flot géodésique soit d'Anosov.

Encore une fois, l'existence de probabilité invariante par toutes les transformations $\rho(\gamma)$ est très restrictive :

ThÉORÈme 5. - Pour toute surface de Riemann compacte $S$ de genre $g \geqslant 2$, l'ensemble des représentations $\rho: \pi_{1}(S) \rightarrow \operatorname{PSL}(2, \mathbb{C})$ ne possèdant aucune probabilité de $\mathbb{C P}^{1}$ invariante par l'action naturelle de l'image $\rho\left(\pi_{1}(S)\right)$, est un ouvert dense dans l'ensemble des représentations.

Rappelons maintenant la notion de mesure de Sinaï-Ruelle-Bowen (SRB) d'une transformation $f: M \rightarrow M$ dans une variété $M$ : c'est une mesure de probabilité borélienne $\mu$ qui est $f$-invariante et telle que

$$
\lim _{n \rightarrow+\infty} \frac{1}{n} \sum_{j=0}^{n-1} \varphi\left(f^{j}(x)\right)=\int \varphi d \mu
$$

pour tout fonction continue $\varphi: M \rightarrow \mathbb{R}$ et pour un ensemble de points $x \in M$ (qu'on appelle le bassin de $\mu$ ) avec mesure de Lebesgue positive dans $M$. Sinaï, Ruelle et Bowen $[25,24,8,7]$ ont montré que si $\Lambda$ est un attracteur hyperbolique d'un difféomorphisme ou d'un flot de classe $C^{2}$ alors il existe une unique mesure SRB dont le support est l'attracteur : c'est un état d'équilibre pour un choix particulier du potentiel. Ceci s'applique, en particulier, aux difféomorphismes et aux flots d'Anosov. 
Proposition 0.2. - Sous les hypothèses du Théorème 3, en supposant de plus que le flot $X$ est de classe $C^{2}$, notons $\mu$ la mesure $S R B$ de $X$.

Alors, si le plus grand exposant, pour $\mu$, du cocycle projectif induit par $Y$ est de multiplicité 1, il existe une unique mesure SRB pour $Y$, elle se projette sur $\mu$, et son bassin a mesure de Lebesgue totale dans $\mathcal{E}$.

Remarquons que, quand $k=2$, l'hypothèse de multiplicité 1 est toujours vérifiée si les exposants sont non-nuls. Pour le flot géodésique ceci reste vrai quand $k=3$, parce que les exposants de Lyapunov du flot et de son inverse sont les mêmes. Le flot $X_{\rho}$ admet alors une unique mesure SRB dont le bassin d'attraction est de mesure de Lebesgue totale dans le fibré unitaire tangent du feuilletage $\mathcal{F}_{\rho}$. (Voir également [4], qui démontre la Proposition 0.2 dans le cadre des surfaces hyperboliques de volume fini, et caractérise la validité du Théorème d'Oseledets dans ce cadre non-compact.) On obtient donc :

COROLlaire 0.3. - Soit $S$ une surface compacte hyperbolique. Il existe un ouvert dense dans l'ensemble des représentations $\rho: \pi_{1}(S) \rightarrow P S L(2, \mathbb{C})$ formé de représentations pour lesquelles le flot géodésique feuilleté $X_{\rho}$ (tangent au feuilles du feuilletage suspension $\mathcal{F}_{\rho}$ ) possède une unique mesure $S R B$, dont le bassin a mesure de Lebesgue totale.

Finalement, [5] montre que, pour les représentations $\rho: \pi_{1}(S) \rightarrow \operatorname{PSL}(2, \mathbb{C})$ dont le cocycle induit a un plus grand exposant de multiplicité 1 , il existe une unique mesure de probabilité $v$ sur la variété $M_{\rho}$ qui décrit le comportement statistique de toutes les feuilles de $\mathcal{F}_{\rho}$ : la mesure de probabilité naturelle portée par de grands disques $D\left(x_{n}, r_{n}\right)$ dans les feuilles de $\mathcal{F}_{\rho}$ converge (faiblement) vers $v$ quand le rayon $r_{n}$ tend vers $+\infty$, indépendamment du centre $x_{n}$ et de la feuille de $\mathcal{F}_{n}$ contenant le disque $D\left(x_{n}, r_{n}\right)$.

Cet article est organisé de la façon suivante. Dans la Section 1 nous obtenons les résultats techniques qui sont à la base de nos théorèmes principaux. Ils sont formulés pour des cocycles, ou bien localement constants ou bien dominés, au dessus d'un sousshift de type fini.

Dans la Section 2 nous traduisons ces résultats dans le cadre des cocycles dominés au-dessus d'une dynamique hyperbolique, pour démontrer les Théorèmes 1 et 2 et la Proposition 0.1 .

Dans la Section 3 nous considérons les cocycles obtenus par relèvement d'un flot d'Anosov, tangent à un feuilletage. Nous réduisons ces systèmes à des cocycles localement constants et, en utilisant des résultats de la Section 1, nous en déduisons le Théorème 3. De plus, nous obtenons la Proposition 0.2.

Dans la Section 4 nous rappelons la notion de suspension d'une représentation dans $\operatorname{PSL}(k, \mathbb{C})$ du groupe fondamental d'une surface, et nous déduisons le Théorème 4 du Théorème 3. Ensuite nous montrons que les exposants de Lyapunov non-nuls sont génériques parmi les représentations dans $\operatorname{PSL}(2, \mathbb{C})$, comme l'affirme le Théorème 5.

\section{Cocycles au-dessus d'un sous-shift de type fini}

Dans cette section nous considérons des cocycles linéaires et projectifs $F: \mathcal{E} \rightarrow \mathcal{E}$ audessus d'un sous-shift de type fini $f: \Sigma_{T} \rightarrow \Sigma_{T}$ bilatère à $n$ symboles associé à une matrice $T \in \mathcal{M}(n, \mathbb{R})$ à coefficients dans $\{0,1\}$. 
Dans ce cadre on peut toujours supposer que le fibré $\pi: \mathcal{E} \rightarrow \Sigma_{T}$ est trivial, c'est à dire que $\mathcal{E}=\Sigma_{T} \times \mathbb{C}^{k}$ (respectivement $\mathcal{E}=\Sigma_{T} \times \mathbb{C P}^{k-1}$ dans le cas projectif). Alors pour chaque cocycle $F: \mathcal{E} \rightarrow \mathcal{E}$ on peut trouver une application $A: \Sigma_{T} \rightarrow S L(k, \mathbb{C})$ (respectivement $A: \Sigma_{T} \rightarrow P S L(k, \mathbb{C})$ ) telle que le cocycle s'écrit sous la forme

$$
F(x, u)=f_{A}(x, u)=(f(x), A(x) u) .
$$

On appelle l'application $f_{A}: \Sigma_{T} \times \mathbb{C}^{k} \rightarrow \Sigma_{T} \times \mathbb{C}^{k}$ (respectivement $f_{A}: \Sigma_{T} \times \mathbb{C P}^{k-1} \rightarrow$ $\Sigma_{T} \times \mathbb{C P}^{k-1}$ ) le cocycle linéaire (respectivement projectif) engendré par $A$.

Pour chaque $n \geqslant 1$, on notera $A^{n}(x)=A\left(f^{n-1}(x)\right) \cdots A(f(x)) \cdot A(x)$ et $A^{-n}(x)=$ $\left(A^{n}\left(f^{-n}(x)\right)\right)^{-1}$.

On dira qu'une application $g: X \rightarrow Y$ entre deux espaces métriques $X$ et $Y$ est $(C, v)$ Hölder continue, pour $C>0$ et $v \in] 0,1]$, si $d\left(f\left(x_{1}\right), f\left(x_{2}\right)\right) \leqslant C d\left(x_{1}, x_{2}\right)^{v}$ pour tout $x_{1}, x_{2}$ dans $X$. On dira que $g$ est $\nu$-Hölder continue s'il existe $C>0$ tel que $g$ soit $(C, v)$-Hölder continue.

\subsection{Sous-shifts de type fini et mesures ayant la propriété de produit local}

Rappelons que $\Sigma_{T}$ est un compact de $\{1, \ldots, n\}^{\mathbb{Z}}$. On munit $\Sigma_{T}$ de la famille de métriques $d_{\theta}$ définies, pour chaque $\theta \in(0,1)$, par

$$
d_{\theta}(x, y)=\theta^{D(x, y)},
$$

où $D(x, y)$ est le plus grand entier non-négatif tel que $x_{j}=y_{j}$ pour tout $|j| \leqslant D(x, y)$ (si $x_{0} \neq y_{0}$ on prend $D(x, y)=0$ ). Ces distances sont toutes équivalentes entre elles, par des homéomorphismes Hölder continus. On peut donc parler de fonction $\varphi: \Sigma_{T} \rightarrow \mathbb{C}$ Hölder continue, la définition ne dépendant pas du choix de $\theta$ (même si les constantes de Hölder en dépendent). On dira qu'une métrique $d$ quelconque est dans la classe Hölder des métriques $d_{\theta}$ si elle est équivalente à ces métriques par des homéomorphismes qui sont Hölder continus, ainsi que leurs inverses.

Pour tout $x=\left(\ldots, x_{-j}, \ldots, x_{0}, x_{1}, \ldots, x_{k}, \ldots\right) \in \Sigma_{T}$ on appelle variété stable locale de $x$ et on note $W_{\text {loc }}^{s}(x)$ l'ensemble des $y=\left(\ldots, y_{-j}, \ldots, y_{0}, y_{1}, \ldots, y_{k}, \ldots\right) \in \Sigma_{T}$ tels que $x_{k}=y_{k}$ pour tout $k \geqslant 0$. On définit de même la variété instable locale $W_{\mathrm{loc}}^{u}(x)$ comme l'ensemble des $y$ tels que $x_{k}=y_{k}$ pour tout $k \leqslant 0$.

On appelle cylindre de $\Sigma_{T}$ tout sous-ensemble donné en prescrivant un nombre fini de coordonnées. Pour tout $j \leqslant 0 \leqslant k$ et toute famille $a_{j}, \ldots, a_{k} \in\{1, \ldots, n\}$ on note $\left[j ; a_{j}, \ldots, a_{k}\right]$ le cylindre donné par $x_{i}=a_{i}$ pout tout $i \in[j, k]$. Un sous-ensemble $E$ de $\left[j ; a_{j}, \ldots, a_{k}\right]$ est appelé $s$-saturé si pour tout $x \in E$ l'ensemble des $y$ tels que $y_{i}=x_{i}$ pour tout $i \geqslant j$, est inclus dans $E$. On définit de même les sous-ensembles $u$-saturés de $\left[j ; a_{j}, \ldots, a_{k}\right]$.

DÉFINITION 1.1. - Une mesure de probabilité $\mu$ sur $\Sigma_{T}$ a la propriété de produit local si pour tout $x \in \Sigma_{T}$ il existe un cylindre $\left[j ; a_{j}, \ldots, a_{k}\right]$ contenant $x$ tel que $\mu(E \cap F)>0$, pour toute partie mesurable s-saturée $E \subset\left[j ; a_{j}, \ldots, a_{k}\right]$ et toute partie mesurable u-saturée $F \subset\left[j ; a_{j}, \ldots, a_{k}\right]$ telles que $\mu(E)>0$ et $\mu(F)>0$. 
La définition revient à dire que la mesure produit $\mu^{u} \times \mu^{s}$ est absolument continue par rapport à $\mu$ dans le cylindre, oú $\mu^{s}$ est la projection de $\mu$ dans l'espace des variétés unstables locales et $\mu^{u}$ est la projection de $\mu$ dans l'espace des variétés stables locales.

Cette propriété est vérifiée par tout état d'équilibre d'un potentiel Hölder continu pour le sous-shift $\left(\Sigma_{T}, f\right)$, voir $[2,12,19]$.

Quitte à raffiner l'alphabet des symboles $\{1, \ldots, n\}$, on peut toujours prendre $j=0=$ $k$ dans la définition; dans la suite nous supposerons que c'est le cas.

Nous commençons par montrer que le support d'une mesure avec la propriété de produit local est toujours un sous-shift de type fini (contenu dans $\Sigma_{T}$ ).

Lemme 1.2. - Soit $\mu$ une mesure de probabilité $f$-invariante dans $\Sigma_{T}$ ayant la propriété de produit local, et soit $N \in \mathcal{M}(n, \mathbb{R})$ la matrice définie par

$$
N_{i, j}=1 \quad \text { si } \mu\left([0 ; i] \cap f^{-1}([0 ; j])\right)>0 \quad \text { et } \quad N_{i, j}=0 \quad \text { si } \mu\left([0 ; i] \cap f^{-1}([0 ; j])\right)=0 \text {. }
$$

Alors le support de $\mu$ cö̈ncide avec le sous-shift de type fini $\Sigma_{N} \subset \Sigma_{T}$ associé à $N$.

Démonstration. - Il nous suffit de montrer que $\mu\left(\left[j ; a_{j}, \ldots, a_{k}\right]\right)>0$ pour tout suite $N$-admissible $a_{j}, \ldots, a_{k}$, c'est à dire, telle que $N_{a_{i}, a_{i+1}}=1$ pour tout $j \leqslant i<k$. De plus, comme $\mu$ est invariante, on peut supposer $j=0$. Nous raisonnons par induction en $k$. Le cas $k=1$ (ou $k=0$ ), est une conséquence immediate de la définition de $N$. Pour le cas général, supposons que $k \geqslant 1$ et que $\mu\left(\left[0 ; a_{0}, \ldots, a_{k}\right]\right)>0$. Alors $f^{k}\left(\left[0 ; a_{0}, \ldots, a_{k}\right]\right)$ est un sous-ensemble $u$-saturé du cylindre $\left[0 ; a_{k}\right]$ avec $\mu$-mesure positive. D'autre part, $\left[0 ; a_{k}\right] \cap f^{-1}\left(\left[0 ; a_{k+1}\right]\right)$ est un sous-ensemble $s$-saturé de $\left[0 ; a_{k}\right]$ aussi avec $\mu$-mesure positive. La propriété de produit local entraine que la $\mu$-mesure de $f^{k}\left(\left[0 ; a_{0}, \ldots, a_{k}\right]\right) \cap f^{-1}\left(\left[0 ; a_{k+1}\right]\right)$ est positive. Comme $\left[0 ; a_{0}, \ldots, a_{k}, a_{k+1}\right]$ est la preimage par $f^{k}$ de ce dernier ensemble, on conclut que sa $\mu$-mesure est positive, comme on l'avait annoncé.

Cela veut dire que, quitte à remplacer $T$ par $N$, on peut supposer que le support de $\mu$ est tout le sous-shift $\Sigma_{T}$. Remarquons que certains symboles $a \in\{1, \ldots, n\}$ peuvent devenir superflus, parce $N_{a, i}=0$ pour tout $i$ ou $N_{i, a}=0$ pour tout $i$ : dans ce cas, on les enlève de l'alphabet, réduisant donc le nombre $n$ des symboles.

Désormais, pour une mesure invariante avec la propriété de produit local, on suppposera toujours qu'elle est de support total.

Voici encore une conséquence directe de la Définition 1.1 :

LEMME 1.3. - Soit $\mu$ une mesure de probabilité dans $\Sigma_{T}$ avec la propriété de produit local. Alors, pour tout ensemble $E \subset[0 ; i]$ de $\mu$-mesure totale dans un cylindre $[0 ; i]$ et pour presque tout point $x \in E$ on a que $E_{x}=\bigcup\left\{W_{\mathrm{loc}}^{u}(y): y \in W_{\mathrm{loc}}^{s}(x) \cap E\right\}$ a aussi $\mu$-mesure totale dans $[0 ; i]$.

Démonstration. - Soit $\mu^{u}$, respectivement $\mu^{s}$, la projection de $(\mu \mid[0 ; i])$ dans l'espace des variétés stables locales, respectivement instables locales, dans $[0 ; i]$. Remarquons que ces espaces peuvent être identifiés à la variété instable, respectivement stable, d'un point fixé quelconque. Supposons que l'ensemble $B$ des points $x$ tels que $\mu^{s}\left(W_{\mathrm{loc}}^{s}(x) \cap E^{c}\right)>0$ ait $\mu$-mesure positive. Comme il s'agit d'un ensemble $s$-saturé, il s'en suit que $\mu^{u}(B)>0$. Alors, intégrant sur l'espace des variétés instables locales, on 
obtient que

$$
\left(\mu^{u} \times \mu^{s}\right)\left(E^{c}\right) \geqslant \int_{B} \mu^{s}\left(W_{\mathrm{loc}}^{s}(x) \cap E^{c}\right) d \mu^{u}(x)>0 .
$$

La propriété de produit local veut dire que le produit $\mu^{u} \times \mu^{s}$ est absolument continu par rapport à $\mu$. Donc, on conclut que $\mu\left(E^{c}\right)$ est positif, ce qui est en contradiction avec l'hypothèse. Ceci montre que $\mu^{s}\left(W_{\text {loc }}^{s}(x) \cap E^{c}\right)=0$ pour $\mu$-presque tout $x \in[0 ; i]$. Pour un tel point $x$ l'ensemble $u$-saturé $E_{x}$ a $\mu$-mesure totale dans le cylindre.

\subsection{Un résultat de Ledrappier sur les cocycles linéaires}

Ce paragraphe est une traduction, dans notre cadre, du résultat de [17, Theorem 1] qui adapte le Théorème de Furstenberg mentionné ci-dessus au cas des produits de matrices dont les tirages ne sont pas indépendants.

On appellera cocycle projectif mesuré la donnée de $\left(\Sigma_{T}, f, \mu, A, f_{A}, m\right)$, où

1. $f: \Sigma_{T} \rightarrow \Sigma_{T}$ est le sous-shift bilatère de type fini à $n$ symboles associé à une matrice $T \in \mathcal{M}(n, \mathbb{R})$ à coefficients dans $\{0,1\}$;

2. $\mu$ est une mesure de probabilité borélienne $f$-invariante ergodique ;

3. $A: \Sigma_{T} \rightarrow S L(k, \mathbb{C})$ est une application continue ;

4. $f_{A}: \Sigma_{T} \times \mathbb{C P}^{k-1} \rightarrow \Sigma_{T} \times \mathbb{C P}^{k-1}$ est le cocycle projectif engendré par $A$ audessus de $f$, et $\pi: \Sigma_{T} \times \mathbb{C P}^{k-1} \rightarrow \Sigma_{T}$ la projection canonique;

5. $m$ est une mesure de probabilité borelienne $f_{A}$-invariante sur $\Sigma_{T} \times \mathbb{C P}^{k-1}$ telle que $\pi_{*}(m)=\mu$.

On définit de même la notion de cocycle linéaire mesuré.

Remarque 1.4. - Si $f_{A}: \Sigma_{T} \times \mathbb{C P}^{k-1} \rightarrow \Sigma_{T} \times \mathbb{C P}^{k-1}$ est continue, pour toute mesure de probabilité $f$-invariante $\mu$ il existe des mesures $f_{A}$-invariantes $m$ se projettant sur $\mu$ : il suffit de prendre pour $m$ un point d'accumulation quelconque, pour la topologie faible, de la suite

$$
\frac{1}{n} \sum_{j=0}^{n-1}\left(f_{A}^{j}\right)_{*}(\mu \times v),
$$

où $v$ est une probabilité quelconque dans $\mathbb{C} \mathbb{P}^{k-1}$.

Nous noterons $\left(m_{x}\right)_{x \in M}$ une désintégration, au sense de Rokhlin [22], de la mesure $m$ le long des fibres $\{x\} \times \mathbb{C P}^{k-1}$. Rappelons que chaque $m_{x}$ est une probabilité conditionnelle de $m$ sur la fibre au-dessus de $x$, et qu'elle est essentiellement uniquement définie : deux désintégrations coïncident $\mu$-presque partout.

Le résultat suivant est un corollaire direct de [17, Theorem 1] :

Corollaire 1.5. - Soit $\left(\Sigma_{T}, f, \mu, A, f_{A}, m\right)$ un cocycle projectif mesuré. Supposons que les exposants de Lyapunov du cocycle $f_{A}$ pour la mesure $\mu$ sont tous nuls. Supposons, de plus, que $A(x), x \in \Sigma_{T}$, ne dépend que de la variété stable locale de $x$, c'est à dire, $A(x)=A(y)$ si $y \in W_{\text {loc }}^{s}(x)$. 
Alors les mesures conditionnelles $m_{x}$ ne dépendent que de la variété stable locale de $x$ : il existe $E \subset \Sigma_{T}$, tel que $\mu(E)=1$ et tel que

$$
m_{x}=m_{y} \quad \text { si } x \in E, \quad y \in E \quad \text { et } \quad y \in W_{\mathrm{loc}}^{s}(x) .
$$

Démonstration. - Notons $\mathcal{B}$ la famille des boréliens de $\Sigma_{T}$ tels que pour tout $B \in \mathcal{B}$ et pour tout $x \in B$ on a $W_{\text {loc }}^{s}(x) \subset B$. La démonstration consiste à voir que le cocycle $A$ et la tribu $\mathcal{B}$ vérifient les hypothèses de [17, Theorem 1] :

Pour tout $x \in \Sigma_{T}$ l'image $f^{-1}\left(W_{\text {loc }}^{s}(x)\right)$ est une union de variétés stables locales. On en déduit que pour tout $B \in \mathcal{B}$ on a $f^{-1}(B) \in \mathcal{B}$. Ceci montre que la tribu $\mathcal{B}$ est décroissante : $f^{-1}(\mathcal{B}) \subset \mathcal{B}$.

Par hypothèse le cocycle $A$ est constant sur les variétés stables locales. L'application $x \mapsto A(x)$ est donc $\mathcal{B}$-mesurable : suivant les notations de [17], la tribu $\sigma(A)$ engendrée par $A$ vérifie $\sigma(A) \subset \mathcal{B}$.

La tribu $\mathcal{B}_{-\infty}$ engendrée par l'union des itérés $f^{n}(\mathcal{B}), n \in \mathbb{Z}$ est la tribu des boréliens de $\Sigma_{T}$. On en déduit que l'application $x \mapsto m_{x}$ est $\mathcal{B}_{-\infty}$-mesurable.

Theorem 1 dans [17] assure alors que l'application $x \mapsto m_{x}$ est $\mathcal{B}$-mesurable. C'est à dire que, modulo un ensemble de mesure 0 pour $\mu$, elle est constante sur les variétés stables locales : c'est ce que le corollaire annonçait.

Remarque 1.6. - Même si le Corollaire 1.5 peut paraître naturel, il faut remarquer que la conclusion est fausse, en général, si l'on enlève l'hypothèse de nullité des exposants de Lyapunov. Par exemple, s'il existe un plus grand exposant de multiplicité 1, la direction correspondante n'est pas, en général, constante le long de variétés stables locales : la mesure $m$ dont les conditionnelles sont les mesures de Dirac aux points représentant cette direction fournit un contre-exemple.

\subsection{Cocycles localement constants}

Dans ce paragraphe nous donnons quelques conséquences du Corollaire 1.5 dans le cas où l'application $x \mapsto A(x)$ est localement constante, et où la mesure $\mu$ possède la propriété de produit local.

Une application $x \mapsto A(x)$ est localement constante si pour tout $x \in \Sigma_{T}$ il existe un cylindre qui le contient et tel la valeur de $A$ est constante sur ce cylindre. Comme avant, quitte à raffiner l'alphabet on peut supposer que ce cylindre est de la forme $[0 ; i]$. Désormais, nous fixons l'alphabet, et nous ne considérons que les cocycles constants sur chaque cylindre $[0 ; i]$.

Corollaire 1.7. - Soit $\left(\Sigma_{T}, f, \mu, A, f_{A}, m\right)$ un cocycle projectif mesuré. On suppose de plus que $\mu$ a la propriété de produit local et que l'application $x \mapsto A(x)$ est constante sur chaque cylindre $[0 ; i]$.

Si les exposants de Lyapunov du cocycle $f_{A}$ par rapport à $\mu$ sont tous nuls, alors l'application $x \mapsto m_{x}$ est constante sur un sous-ensemble de $\mu$-mesure totale de chaque cylindre $[0 ; i]$.

Démonstration. - L'hypothèse implique que $A$ est constante sur les variétés stables ou instables locales; on peut donc appliquer à $f_{A}$ et à $f_{A}^{-1}$ le Corollaire 1.5. On conclut que 
les mesures conditionnelles $m_{x}$ sont constantes $\mu$-presque partout le long des variétés stables et instables locales. En utilisant la propriété de produit local on obtient que $m_{x}$ est constante $\mu$-presque partout sur les cylindres $\left[0 ; a_{0}\right]:$ sinon il existerait dans $\left[0 ; a_{0}\right]$ deux ensembles mesurables $E_{0}$ et $F_{0}$ de mesure positive et une fonction continue $\phi: \mathbb{C P}^{k-1} \rightarrow \mathbb{R}$ telle que $\int \phi d m_{x}-\int \phi d m_{y}>0$ pour tout $x \in E_{0}$ et tout $y \in F_{0}$. Soit $E$ le saturé de $E_{0}$ par les variétés stables locales et $F$ le saturé de $F_{0}$ par les variétés instables locales. La propriété de produit local de $\mu$ assure que $\mu(E \cap F)>0$. D'autre part, pour $\mu$-presque tout point $z \in E \cap F$ il existe $x \in E_{0}$ et $y \in F_{0}$ tels que $\int \phi d m_{z}=\int \phi d m_{x}$ et $\int \phi d m_{z}=\int \phi d m_{y}$, contredisant le choix de $E_{0}$ et $F_{0}$.

Voici une version globale du Corollaire 1.7 :

COROllaire 1.8. - Sous les hypothèses du Corollaire 1.7, il existe un changement de coordonnées linéaires dans les fibres, constant sur chaque cylindre [0; i], de façon que dans les nouvelles coordonnées la mesure $m_{x}$ soit constante $\mu$-presque partout. Notons $m_{0}$ cette probabilité. De plus, $\hat{A}(x)$ preserve $m_{0}$ pour tout $x \in \Sigma_{T}$, où $\hat{A}(x)$ est l'expression du cocycle dans les nouvelles coordonnées.

Démonstration. - D'après le Corollaire 1.5, les mesures conditionnelles $m_{x}$ sont constantes $\mu$-presque partout sur chaque cylindre $[0 ; i]$. Soit $m_{i}$ la valeur correspondante. Comme $\mu$ est ergodique, pour tout $j \in\{1, \ldots, n\}$ il existe $l$ tel que $\mu([0 ; 1] \cap$ $\left.f^{-l}([0 ; j])\right)>0$. On considère $j>1$ et on choisit $l$ minimal avec cette propriété. Pour $\mu$-presque tout $x \in[0 ; 1] \cap f^{-l}([0 ; j])$ on a : $A^{l}(x)_{*}\left(m_{1}\right)=m_{j}$. Ceci est une conséquence de l'invariance de la mesure $m$ et de l'unicité $\mu$-presque partout des mesures conditionnelles.

On choisit un point $x_{j} \in[0 ; 1] \cap f^{-l}([0 ; j])$ satisfaisant $m_{x_{j}}=m_{1}$ et $m_{f^{l}\left(x_{j}\right)}=m_{j}$, et tel que $A^{l}\left(x_{j}\right)_{*}\left(m_{1}\right)=m_{j}$. On change les coordonnées dans les fibres de $[0, j]$ par une application linéaire constante sur ce cylindre, de façon que dans ces coordonnées $A^{l}\left(x_{j}\right)$ soit la matrice de l'identité. Dans ces coordonnées, la probabilité $m_{j}$ est égale à $m_{1}$. Nous avons donc obtenu un changement de coordonnées linéaires dans les fibres, constant sur les cylindres, dans lequel les probabilités conditionnelles $m_{x}$ sont égales à $m_{1}$ pour $\mu$-presque tout $x \in \Sigma_{T}$.

Comme on l'avait remarqué ci-dessus les probabilités conditionnelles sont préservées $\mu$-presque partout par l'action de $A(x)$. Nous avons donc $A(x)_{*}\left(m_{1}\right)=m_{1}$ pour $\mu$ presque tout point. Les changements de coordonnées ayant été choisis constants dans les cylindres, le cocycle reste constant sur chaque $[0 ; i]$, donc continu, après changement de coordonnées. Par continuité, la relation $A(x)_{*}\left(m_{1}\right)=m_{1}$ reste valable sur tout le support de la mesure, ce qui conclut.

Ceci veut dire, en particulier, que $m_{0}$ est invariante par $A^{p}(x)$ pour tout point périodique $x$ de période $p \geqslant 1$. Cela nous permet de montrer, dans le prochain corollaire, que les cocycles localement constants génériques ont des exposants de Lyapunov nonnuls.

On munit de la topologie $C^{0}$ l'espace des applications $A: \Sigma_{T} \rightarrow S L(k, \mathbb{C})$ constantes sur chaque cylindre $[0 ; i]$. Pour une telle application, on note $A_{i}$ sa valeur sur chaque $[0 ; i]$. 
Corollaire 1.9.- Soit $f: \Sigma_{T} \rightarrow \Sigma_{T}$ un sous-shift de type fini, muni d'une mesure de probabilité ergodique avec structure de produit local.

Il existe un sous-ensemble ouvert dense d'applications $A: \Sigma_{T} \rightarrow S L(k, \mathbb{C})$ constantes sur chaque cylindre $[0 ; i]$ telles que le cocycle $f_{A}$ engendré par A possède des exposants de Lyapunov non-nuls.

Démonstration. - Le cas où $\mu$ est une mesure de Dirac sur une orbite périodique est une simple conséquence du fait que les matrices génériques (ouvert dense) en dimension quelconque sont hyperboliques. On va donc supposer que $\Sigma_{T}=\operatorname{supp}(\mu)$ est infini.

Alors, on peut choisir un point périodique $p \in \Sigma_{T}$ et un point homocline $q \in \Sigma_{T}$ associé à $p$ : c'est à dire, un point non-périodique dont l'orbite passe, à la fois, par la variété stable locale et la variété instable locale de $p$. On va supposer d'abord que $p$ est un point fixe de $f$ et ensuite on expliquera comment l'argument s'adapte au cas général. Écrivons $p=\left(\ldots, a_{0}, \ldots\right)$ et $q=\left(\ldots, a_{0}, \ldots, a_{0}, a_{1}, \ldots a_{s}, a_{0}, \ldots, a_{0}, \ldots\right)$, avec $a_{i} \in\{1, \ldots, n\}$. Nous supposons que $q$ a été choisi de façon que $s$ soit minimal. Remarquons que dans ce cas les symboles $a_{0}, a_{1}, \ldots, a_{s}$ sont nécéssairement tous différents.

Supposons que les exposants de Lyapunov de $A$ pour $\mu$ sont tous nuls. On commence par remplacer $A_{a_{0}}$ par une matrice $\tilde{A}_{a_{0}}$ arbitrairement proche, telle que ses valeurs propres soient toutes de multiplicité 1 et de modules différents. Alors $\tilde{A}_{a_{0}}$ n'admet comme probabilités invariantes dans $\mathbb{C P}^{k-1}$ que les combinaisons convexes des mesures de Dirac $\delta_{\xi_{l}}$ correspondant aux directions propres $\xi_{1}, \ldots, \xi_{k}$. Ensuite, on remplace la valeur $A_{a_{s}}$ de $A$ sur le cylindre $\left[0 ; a_{s}\right.$ ] par une matrice $\tilde{A}_{a_{s}}$, arbitrairement proche de $A_{a_{s}}$, telle que l'ensemble

$$
\left\{\tilde{A}_{a_{s}} A_{a_{s-1}} \cdots A_{a_{1}} \cdot \xi_{l}: l=1, \ldots, k\right\}
$$

des images des directions propres soit disjoint de l'ensemble $\left\{\xi_{l}: l=1, \ldots, k\right\}$ des directions propres elles-mêmes. De cette façon, en gardant les valeurs de $A$ sur tous les autres cylindres, on obtient une nouvelle application $B$ de $\Sigma_{T}$ dans $\operatorname{SL}(k, \mathbb{C})$, arbitrairement proche de $A$ et qui est aussi constante sur chaque cylindre $[0 ; i]$. On note $f_{B}$ le cocycle correspondant. On va montrer, par l'absurde, que $f_{B}$ a au moins un exposant de Lyapunov non-nul.

En effet, d'après la Remarque 1.4 , on peut toujours choisir une probabilité $f_{B^{-}}$invariante $m$ se projettant sur $\mu$. D'après le Corollaire 1.9, il existe un changement de coordonnées constant sur les cylindres dans le quel les mesures conditionelles $m_{x}$ de $m$ peuvent être choisies constantes (partout). De plus, si on note $m_{0}$ cette constante et $\hat{B}(x)$ l'expression de $B$ dans les nouvelles coordonnées, alors $m_{0}$ est invariante par $\hat{B}(x)$ pour tout point $x$. En particulier, $m_{0}$ est une probabilité invariante par $\hat{B}(p)$ (qui est conjugué a $\left.\tilde{A}_{a_{0}}\right)$. Donc, elle doit être une combinaison convexe des mesures de Dirac correspondant aux directions propres de $\hat{B}(p)$. D' autre part, $m_{0}$ doit être invariante aussi par $\hat{B}\left(f^{n}(q)\right)$ pour tout $n \in \mathbb{Z}$. Ceci contredit le choix de $\tilde{A}_{a_{s}}$, ce qui démontre l'énoncé de densité.

Maintenant, soit $C: \Sigma_{T} \rightarrow S L(k, \mathbb{C})$ une application quelconque, constante sur les cylindres et suffisament proche de $B$. Alors, les valeurs propres de $C_{a_{0}}$ sont aussi de multiplicité 1 , et leur modules sont encore tous différents. De plus, l'ensemble des images de ses directions propres par la transformation $C_{a_{s}} \cdots C_{a_{1}}$ reste disjoint de 
l'ensemble des directions propres. Cela veut dire que l'argument précédent reste valable pour $C$ : les exposants de Lyapunov du cocycle engendré par $C$ ne sont pas tous nuls. Ceci montre que l'ensemble des cocycles dont les exposants de Lyapunov ne sont pas tous nuls contient un sous-ensemble ouvert dense de l'espace des cocycles constants sur les $[0 ; i]$.

Finalement, supposons que $\Sigma_{T}$ ne contient pas de point fixe. Comme on suppose aussi que $\Sigma_{T}$ n'est pas juste une orbite périodique, il existe au moins un point périodique dont l'itinéraire n'utilise pas tous les symboles de l'alphabet. De plus, il existe un point homocline $q$ associé a $p$ qu'on peut choisir de façon que la partie non-périodique de son itinéraire (un interval) n'utilise pas les symboles qui sont dans l'itinéraire de $p$. L'argument se termine en utisant les mêmes idées qu'avant.

Remarque 1.10. - L'énoncé d'ouverture est probablement faux si l'on considère l'espace de tous les cocycles localement constants (sans fixer la partition sur laquelle l'application $A$ est constante).

L'ensemble ouvert dense que nous avons construit dans la preuve du Corollaire 1.9 est indépendent de l'état d'équibre que l'on considère.

\subsection{Cocycles $s$-dominés : cadre abstrait}

Les résultats dans ce paragraphe sont en rapport avec la théorie de la cohomologie pour les cocycles dans des groupes non-abéliens au dessus d'un système hyperbolique ; voir par exemple [21,15].

DÉFINITION 1.11. - Soit $\left(\Sigma_{T}, f, A, f_{A}\right)$ un sous-shift de type fini muni d'un cocycle linéaire ou projectif. On dira que le cocycle $f_{A}$ est dominé dans la direction stable (ou, simplement, $s$-dominé) s'il existe une métrique d sur $\Sigma_{T}$, et il existe des constantes $\tau<1, v \in] 0,1], N \in \mathbb{N}$ et $\theta_{1}, \ldots, \theta_{n}<1$ telles que :

1. $d\left(f^{N}(x), f^{N}(y)\right) \leqslant \theta_{i} d(x, y)$ pour tous $x, y \in[0 ; i]$ tels que $y \in W_{\mathrm{loc}}^{s}(x)$;

2. L'application $x \mapsto A^{N}(x)$ est $v$-Hölder continue par rapport à $d$;

3. $\left\|A^{N}(x)\right\|\left\|A^{N}(x)^{-1}\right\| \theta_{i}^{v}<\tau$ pour tout $x \in[0 ; i]$.

On définit de façon analogue la notion de cocycle dominé dans la direction instable (ou $u$-dominé). Quitte à remplacer $f \operatorname{par} f^{N}$, on peut supposer que $N=1$, et nous le ferons désormais.

Remarquons que la troisième condition est robuste pour les $C^{0}$ perturbations des applications Hölder continues : l'ensemble des cocycles s-dominés est ouvert pour la $C^{0}$ topologie dans l'espace des cocycles $v$-Hölder continus (par rapport à une distance d fixée) avec v fixé.

Lemme 1.12. - Soit $\left(\Sigma_{T}, f, A, f_{A}\right)$ un sous-shift de type fini muni d'un cocycle linéaire ou projectif dominé dans la direction stable. Alors il existe une famille continue bornée de transformations projectives inversibles $\phi(x, y): \mathbb{C P}^{k-1} \rightarrow \mathbb{C P}^{k-1}$, définies pour tout $x, y \in \Sigma_{T}$ tels que $y \in W_{\mathrm{loc}}^{s}(x)$, satisfaisant

1. $\phi(x, x)=$ id et $\phi(y, z) \cdot \phi(x, y)=\phi(x, z)$;

2. $\phi(f(x), f(y))^{-1} \cdot A(y) \cdot \phi(x, y)=A(x)$;

pour tout $x, y, z \in \Sigma_{T}$ dans une même variété stable locale. 
Démonstration. - Considerons $x, y$ dans une même variété stable locale et définissons

$$
\phi_{n}(x, y)=A^{n}(y)^{-1} \cdot A^{n}(x)
$$

pour chaque $n \geqslant 1$. Rappelons que $d\left(f^{n}(x), f^{n}(y)\right) \leqslant \theta_{a_{0}} \cdots \theta_{a_{n-1}} d(x, y)$ où $a_{0}, \ldots, a_{n-1}$ sont determinés par $x, y \in\left[0 ; a_{0}, \ldots, a_{n}\right]$. Rappelons aussi qu'il existe $C_{1}>0$ tel que $\|A(\xi)-A(\eta)\| \leqslant C_{1} d(\xi, \eta)^{v}$ pour tout $\xi, \eta \in \Sigma_{T}$. Nous allons montrer que la suite $\phi_{n}$ est uniformément convergente. En effet,

$$
\phi_{n+1}(x, y)-\phi_{n}(x, y)=A^{n}(y)^{-1} A\left(f^{n}(y)\right)^{-1}\left[A\left(f^{n}(x)\right)-A\left(f^{n}(y)\right)\right] A^{n}(x)
$$

et donc

$$
\left\|\phi_{n+1}(x, y)-\phi_{n}(x, y)\right\| \leqslant C_{1} d(x, y)^{v}\left\|A\left(f^{n}(y)\right)^{-1}\right\| \prod_{j=0}^{n-1}\left\|A\left(f^{j}(y)\right)^{-1}\right\|\left\|A\left(f^{j}(x)\right)\right\| \theta_{a_{j}}^{v} .
$$

Fixons $\tau<\hat{\tau}<1$. Comme $A$ est uniformément continue, et que des points dans une même variété stable locale sont uniformément asymptotiques, la dernière condition dans la Définition 1.11 nous permet de trouver $j_{0} \geqslant 1$, indépendent de $x$ et $y$, tel que

$$
\left\|A\left(f^{j}(y)\right)^{-1}\right\|\left\|A\left(f^{j}(x)\right)\right\| \theta_{a_{j}}^{v}<\hat{\tau}
$$

pour tout $j \geqslant j_{0}$. L'inégalité précédente implique donc qu'il existe des constantes $C_{2}$ et $C_{3}$ telles que

$$
\left\|\phi_{n+1}(x, y)-\phi_{n}(x, y)\right\| \leqslant C_{2} \hat{\tau}^{n} d(x, y)^{v} \leqslant C_{3} \hat{\tau}^{n} .
$$

Ceci implique que $\phi$ est une suite de Cauchy, uniformément en $(x, y)$. Elle est donc uniformément convergente, comme on l'avait annoncé. On notera $\phi(x, y)$ la limite, dans l'espace des transformations linéaires de $\mathbb{C}^{n}$. Il s'agit d'une application (Hölder) continue et donc bornée : l'ensemble des paires de points dans une même variété stable locale est compacte.

On vérifie à présent les propriétés annoncées. Remarquons qu'on a $\phi_{n}(y, z)$. $\phi_{n}(x, y)=\phi_{n}(x, z)$ et $\phi_{n}(x, x)=$ id pour tout $n \geqslant 1$ et tout triplet de points $x, y, z$ appartenant à une même variété stable locale. Ceci montre l'item 1. Il s'en suit aussi que $\phi(x, y)$ est inversible, d'inverse $\phi(y, x)$. Elle définit donc bien une transformation projective de $\mathbb{C P}^{k-1}$, qu' on note encore $\phi(x, y)$. Finalement, la définition de $\phi_{n}(x, y)$ entraîne la relation $\phi_{n+1}(x, y)=A(y)^{-1} \cdot \phi_{n}(f(x), f(y)) \cdot A(x)$. En passant à la limite quand $n \rightarrow \infty$, on obtient l'item 2 du lemme.

Remarque 1.13. - En sommant les inégalités (7) sur tous les $n \geqslant l$ on obtient :

$$
\left\|\phi(x, y)-\phi_{l}(x, y)\right\| \leqslant C_{4} \hat{\tau}^{l} d(x, y)^{v}
$$

pour tout $l \geqslant 1$ et tout $(x, y)$ dans le domaine de définition de $\phi$, avec $C_{4}=C_{2} \sum_{j=0}^{\infty} \hat{\tau}^{j}$. Ceci reste vrai pour $l=0$, si on définit $\phi_{0}=\mathrm{id}:\|\phi(x, y)-\mathrm{id}\| \leqslant C_{4} d(x, y)^{\nu}$. 
On munit l'ensemble des applications $(C, v)$-Hölder continues, avec $C>0$ et $v \in$ ]0, 1] fixés, de la $C^{0}$ topologie.

LEMME 1.14. - La famille d'applications $\phi$ construite dans le Lemme 1.12 varie continûment avec l'application A, par rapport à la $C^{0}$ topologie dans l'ensemble des applications $(C, v)$-Hölder continues, avec $C>0$ et $v \in] 0,1]$ fixés.

Démonstration. - Les constantes $C_{2}$ et $C_{3}$ dans l'inégalité (7) ne dépendent que de la norme de $A$ et de l'ordre $j_{0}$ à partir duquel l'inegalité (6) est vraie, et $j_{0}$ ne dépend que d'un module de continuité de l'application $A$. Comme nous fixons les constantes de Hölder, il s'en suit que $j_{0}$ peut être choisi constant dans le voisinage de toute application $A$, et de même pour $C_{2}$ et $C_{3}$. On en déduit que la convergence est (localement) uniforme aussi en $A$, donc la limite $\phi$ dépend continûment de $A$.

Corollaire 1.15. - Dans les conditions du Lemme 1.12, le cocycle $f_{A}$ est conjugué à un cocycle constant sur des variétés stables locales : il existe une transformation continue $h: \Sigma_{T} \times \mathbb{C P}^{k-1} \rightarrow \Sigma_{T} \times \mathbb{C P}^{k-1}$ se projetant sur l'identité de $\Sigma_{T}$ et projective dans les fibres, telle que le cocycle $h^{-1} \circ f_{A} \circ h$ est constant sur toute variété stable locale.

Démonstration. - Pour chaque $i$ dans $\{1, \ldots, n\}$ on fixe une variété instable locale $W_{i}^{u}$ dans le cylindre $[0 ; i]$. Pour chaque $y$ dans le cylindre il existe un unique $x$ appartenant à l'intersection de $W_{\text {loc }}^{s}(y)$ avec $W_{i}^{u}$. Avec ces notations, on définit $h(y, \xi)=$ $(y, \phi(x, y) \xi)$. Alors, en utilisant l'item 2 du lemme,

$$
\begin{aligned}
h^{-1} \circ f_{A} \circ h(y, \xi) & =h^{-1} \circ f_{A}(y, \phi(y, x) \xi)=h^{-1}(f(y), A(y) \phi(x, y) \xi) \\
& =h^{-1}(f(y), \phi(f(x), f(y)) A(x) \xi) .
\end{aligned}
$$

Notons $x_{1}$ le point d'intersection entre la variété stable locale de $f(x)$ et la variété instable $W_{j}^{u}$ correspondant au cylindre $[0 ; j]$ qui contient $f(x)$. La définition de $h$ donne donc que

$$
\begin{aligned}
h^{-1} \circ f_{A} \circ h(y, \xi) & =\left(f(y), \phi\left(f(y), x_{1}\right) \phi(f(x), f(y)) A(x) \xi\right) \\
& =\left(f(y), \phi\left(f(x), x_{1}\right) A(x) \xi\right) .
\end{aligned}
$$

Ceci démontre que le cocycle conjugué est bien constant sur la variété stable locale de tout point $x$.

Dans la suite on notera $h(y, \xi)=\left(y, h_{y}(\xi)\right)$.

Proposition 1.16. - Soit $\left(\Sigma_{T}, f, \mu, A, f_{A}, m\right)$ un cocycle projectif mesuré tel que $f_{A}$ est dominé dans la direction stable.

Si les exposants de Lyapunov du cocycle sont nuls, il existe un ensemble $E \subset \Sigma_{T}$ avec $\mu(E)=1$ tel que $\phi(x, y)_{*} m_{x}=m_{y}$ pour tout $x, y \in E$ appartenant à une même variété stable locale.

Démonstration. - On considère le cocycle conjugué $\tilde{f}_{A}=h^{-1} \circ f_{A} \circ h$ donné par le Corollaire 1.15. On considère aussi la mesure $\tilde{f}_{A}$-invariante $\tilde{m}=h_{*} m$. Comme l'application $\phi(\cdot, \cdot)$ est bornée, le cocycle $\tilde{f}_{A}$ vérifie toujours les conditions d'intégrabilité du Théorème d'Oseledets, et ses exposants de Lyapunov pour la mesure $\mu$ sont les mêmes 
que ceux de $f_{A}$, c'est à dire, nuls. D'après le Corollaire 1.7, les mesures conditionnelles $\tilde{m}_{x}$ de $\tilde{m}$ sont constantes sur les variétés stables locales, sur un ensemble $E$ de mesure $\mu(E)=1$. En remarquant que $\tilde{m}_{y}=\left(h_{y}\right)_{*} m_{y}$, on obtient que $\left(h_{y}\right)_{*} m_{y}=\left(h_{x}\right)_{*} m_{x}$, c'est à dire, $m_{y}=\left(h_{y}^{-1} \cdot h_{x}\right)_{*} m_{x}$ pour tout $x, y \in E$ dans une même variété stable locale. Par définition de $h$, on a $h_{y}^{-1} \cdot h_{x}=\phi(x, y)$, ce qui démontre la proposition.

\subsection{Cocycles dominés : cadre abstrait}

On dit qu'un cocycle est dominé s'il est à la fois $s$ - et $u$-dominé.

Lemme 1.17. - Soit $\left(\Sigma_{T}, f, \mu, A, f_{A}, m\right)$ un cocycle projectif mesuré. On suppose que $\mu$ a la propriété de produit local, que le cocycle $f_{A}$ est dominé et que ses exposants de Lyapunov pour $\mu$ sont tous nuls.

Alors il existe un changement de coordonnées linéaires dans les fibres, continu, tel que dans les nouvelles coordonnées la mesure conditionnelle $m_{x}$ est $\mu$-presque partout constante sur chaque cylindre $[0 ; i]$.

Démonstration. - On considère les familles continues $\phi^{s}(x, y)$ et $\phi^{u}(x, z)$ construites, respectivement, pour $f_{A}$ et pour $f_{A}^{-1}$, au Lemme 1.12 et au Corollaire 1.15. Rappelons que ces transformations sont définies pour tout point $x \in \Sigma_{T}$ et pour tout $y \in W_{\text {loc }}^{s}(x)$ et tout $z \in W_{\text {loc }}^{u}(x)$. Pour toute paire $(x, z)$ de points dans un même cylindre, il existe un seul $y$ dans le cylindre, tel que $y \in W_{\mathrm{loc}}^{s}(x)$ et $z \in W_{\mathrm{loc}}^{u}(y)$. Ceci nous permet de définir $\psi(x, z)=\phi^{u}(y, z) \cdot \phi^{s}(x, y)$.

Notons $E^{s} \subset[0 ; i]$ un sous-ensemble de $\mu$-mesure totale (donné par la Proposition 1.16) tel que $m_{y}=\phi^{s}(x, y)_{*}\left(m_{x}\right)$ pour tout $x, y \in E^{s}$ appartenant à la même variété stable locale. De même, soit $E^{u} \subset[0 ; i]$ un sous-ensemble de $\mu$-mesure totale tel que $m_{z}=\phi^{u}(y, z)_{*}\left(m_{y}\right)$ pour tout $y, z \in E^{u}$ appartenant à la même variété instable locale. On note $E=E^{u} \cap E^{s}$ : c'est un sous-ensemble de $[0 ; i]$ de $\mu$-mesure totale.

D'après le Lemme 1.3 il existe donc $x_{i} \in[0 ; i]$ tel que $E_{1}=E \cap \bigcup\left\{W_{\text {loc }}^{u}(y): y \in\right.$ $\left.W_{\text {loc }}^{s}\left(x_{i}\right) \cap E\right\}$ soit de mesure totale dans $\Sigma_{T}$. L'ensemble des points $x_{i}$ avec cette propriété est même de $\mu$-mesure totale. Remarquons que, par définition, pour tout $z \in E_{1}$ il existe $y \in E_{1} \cap W_{\text {loc }}^{s}\left(x_{i}\right)$ tel que $z \in W_{\text {loc }}^{u}(y)$. On en déduit que $m_{z}=$ $\phi^{u}(y, z) \cdot \phi^{s}\left(x_{i}, y\right)_{*}\left(m_{x_{i}}\right)=\psi\left(x_{i}, z\right)_{*}\left(m_{x_{i}}\right)$. Les applications $\psi\left(x_{i}, \cdot\right)$ ainsi construites sont le changement de coordonnées annoncé.

Corollaire 1.18. - Dans les conditions du Lemme 1.17, il existe un changement de coordonnées linéaire dans les fibres, continu, tel que dans les nouvelles coordonnées la mesure conditionnelle $m_{x}$ est $\mu$-presque partout constante.

Notons $m_{0}$ cette probabilité de $\mathbb{C} \mathbb{P}^{k-1}$. Pour tout $x \in \Sigma_{T}$, la matrice $\hat{A}(x)$ préserve $m_{0}$, où $\hat{A}(x)$ est la valeur du cocycle en $x$ dans les nouvelles coordonnées.

Démonstration. - D'après le Lemme 1.17 on peut supposer que les mesures conditionnelles $m_{x}$ sont constantes $\mu$-presque partout sur chaque cylindre $[0 ; i]$. Soit $m_{i}$ la valeur correspondante. Comme $\mu$ est ergodique, pour tout $j \in\{1, \ldots, n\}$ il existe $l$ tel que $\mu\left([0 ; 1] \cap f^{-l}([0 ; j])\right)>0$. On considère $j>1$ et on choisit $l$ minimal avec cette propriété. Pour $\mu$-presque tout $x \in[0 ; 1] \cap f^{-l}([0 ; j])$ on a: $A^{l}(x)_{*}\left(m_{1}\right)=m_{j}$. Ceci est une conséquence de l'invariance de la mesure $m$ et de l'unicité $\mu$-presque partout des mesures conditionnelles. 
On choisit un point $x_{j} \in[0 ; 1] \cap f^{-l}([0 ; j])$ satisfaisant $m_{x_{j}}=m_{1}$ et $m_{f^{l}\left(x_{j}\right)}=$ $m_{j}$, et tel que $A^{l}\left(x_{j}\right)_{*}\left(m_{1}\right)=m_{j}$. On change les coordonnées dans les fibres de $[0, j]$ par une application linéaire constante sur ce cylindre, de façon que dans les nouvelles coordonnées $A^{l}\left(x_{j}\right)$ devienne la matrice de l'identité. Dans ces coordonnées, la probabilité $m_{j}$ est égale à $m_{1}$. Nous avons donc obtenu un changement de coordonnées linéaires dans les fibres, continu, dans lequel les probabilités conditionnelles $m_{x}$ sont $\mu$ presque partout égales à $m_{1}$.

Comme on l'avait remarqué ci-dessus les probabilités conditionnelles sont préservées $\mu$-presque partout par l'action de $A(x)$. Nous avons donc $A(x)_{*}\left(m_{1}\right)=m_{1}$ pour $\mu$ presque tout point. Les changements de coordonnées ayant été choisis de façon continue, le cocycle reste continu après changement de coordonnées, la relation $A(x)_{*}\left(m_{1}\right)=m_{1}$ reste valable sur tout le support de la mesure, ce qui conclut.

Le même genre d'arguments nous donne aussi le

THÉORÈME 6. - Soit $\left(\Sigma_{T}, f, \mu, A, f_{A}, m\right)$ un cocycle projectif mesuré. On suppose que $\mu$ a la propriété de produit local, que le cocycle $f_{A}$ est dominé et que ses exposants de Lyapunov pour $\mu$ sont tous nuls.

Alors il existe une désintegration $\left(\bar{m}_{x}\right)_{x}$ de la mesure $m$ telle que

- $\bar{m}_{x}$ varie continûment avec le point $x \in \Sigma_{T}$;

- $A(x)_{*} \bar{m}_{x}=\bar{m}_{f(x)}$ pour tout $x \in \Sigma_{T}$;

- $\psi(x, y)_{*} \bar{m}_{x}=\bar{m}_{y}$ pour tout $x$ et $y$ appartenant à un même cylindre $[0 ; i]$.

Démonstration. - Pour chaque $i$ nous fixons $x_{i} \in[0 ; i]$ comme dans le Lemme 1.17, et nous définissons $\bar{m}_{x}=\psi\left(x_{i}, x\right)_{*} m_{x_{i}}$ pour tout $x$ dans ce cylindre. Comme $\psi$ est une fonction continue, il est clair que $\bar{m}_{x}$ dépend continûment de $x$. De plus, $\bar{m}_{x}=m_{x}$ pour $\mu$-presque tout $x$. Donc, la famille $\left(\bar{m}_{x}\right)_{x}$ est encore une désintégration de la mesure $f_{A}$-invariante $m$ et, par conséquence, on a $A(x)_{*} \bar{m}_{x}=\bar{m}_{f(x)}$ pour $\mu$-presque tout $x$. Par continuité, ceci reste vrai pour tout point $x \in \Sigma_{T}=\operatorname{supp}(\mu)$. De même, comme on l'a vu au Lemme 1.17, il existe un ensemble de mesure totale de points $x_{i} \in[0 ; i]$ tels que pour $\mu$-presque tout $z \in[0 ; i]$ on a $\psi\left(x_{i}, z\right)_{*} \bar{m}_{x_{i}}=\bar{m}_{z}$. Par continuité de $\psi$ et de la famille $\bar{m}_{x}$, cela reste vrai pour toute paire $\left(x_{i}, z\right)$ de points dans un même cylindre.

Finalement, on peut montrer que les cocycles avec exposants de Lyapunov non-nuls sont génériques parmi les cocycles dominés au-dessus d'un sous-shift de type fini.

ThÉORÈme 7. - Pour chaque $C>0$ et $v \in] 0,1]$, il existe un sous-ensemble ouvert dense pour la topologie $C^{0}$ dans l'espace des applications $(C, v)$-Hölder continues $x \rightarrow A(x)$ engendrant un cocycle dominé $f_{A}$ au-dessus d'un shift $f: \Sigma_{T} \rightarrow \Sigma_{T}$, telles que les exposants de Lyapunov du cocycle engendré ne sonts pas tous nuls, pour toute mesure de probabilité $f$-invariante et $f$-ergodique avec la propriété de produit local.

Démonstration. $-\mathrm{Si} \Sigma_{T}$ est réduit à une orbite périodique, l'ennoncé est une conséquence directe du fait que les matrices génériques sont hyperboliques. Nous considerons donc le cas oú $\Sigma_{T}$ contient aussi des points homoclines $q$ associés à un point périodique quelconque $p$. Supposons que $p$ est un point fixe, le cas périodique est analogue. Nous choisissons $q$ comme dans la preuve du Corollaire $1.9:$ il existe $l \geqslant 1$ tel que $q \in W_{\text {loc }}^{u}(p), f^{l}(q) \in W_{\text {loc }}^{s}(p)$, et aucun itéré $f^{i}(q), 0<i<l$, n'est dans le cylindre $\left[0 ; a_{0}\right]$ qui contient $p$. 
L'ensemble ouvert dense dans l'ennoncé du théorème sera donné par deux conditions sur l'application $A$, que nous ennonçons dans la suite. Premièrement, on suppose que $A(p)$ n'a que des valeurs propres de multiplicité 1 et modules tous différents, ce qui est vrai pour un ensemble ouvert dense d'applications. Soient $\xi_{j}, 1 \leqslant j \leqslant k$ les points de $\mathbb{C P}^{k-1}$ représentant les directions propres de $A(p)$.

Dans la deuxième condition, on utilise la famille de transformations $\psi(x, z)=$ $\phi^{u}(y, z) \cdot \phi^{s}(x, y)$ construite dans la démonstration du Lemme 1.17 : on demande que

$$
\left\{A^{l}(q) \psi(p, q) \xi_{j}: 1 \leqslant j \leqslant k\right\} \cap\left\{\psi\left(p, f^{l}(q)\right) \xi_{j}: 1 \leqslant j \leqslant k\right\}=\emptyset .
$$

Les directions propres $\xi_{j}$ dépendent continûment de l'application $A$ et, d'après le Lemme 1.14 , le même est vrai pour les transformations $\psi$. On en déduit que la condition (8) est ouverte.

Remarquons que, pour tout point $z$ dans la variété stable locale de $p, \psi(p, z)=$ $\phi^{s}(p, z)$ ne dépend que de la restriction de $A$ à la variété stable locale de $p$. De même, pour tout $z$ dans la variété instable locale de $p, \psi(p, z)=\phi^{u}(p, z)$ ne dépend que de la restriction de $A$ à la variété instable locale de $p$. En particulier, $\psi(p, q)$ et $\psi\left(p, f^{l}(q)\right)$ ne change pas si l'on modifie le cocycle en dehors du cylindre $\left[0 ; a_{0}\right]$. On peut donc perturber toute application $A$ telle que les valeurs propres de $A(p)$ soient de multiplicité 1 , dans un voisinage de $f^{l-1}(q)$ disjoint de $\left[0 ; a_{0}\right]$, de façon à avoir (8). Ceci montre que la condition (8) est aussi dense.

Soit $A$ une application satisfaisant ces deux conditions et engendrant un cocyle dominé $f_{A}$. Nous allons montrer que les exposants de Lyapunov de $f_{A}$ ne sont pas tous nuls, pour n'importe quelle mesure de probabilité $f$-invariante et ergodique avec la propriété de produit local. Cela conclura la démonstration du théorème.

En effet, supposons que les exposants de Lyapunov de $f_{A}$ pour une telle mesure $\mu$ sont tous nuls. Alors, d'après le Théorème 6 , il existe une famille continue de mesures de probabilité $\left(\bar{m}_{x}\right)_{x}$ qui sont invariantes par l'action du cocycle, c'est à dire que

$$
A(x)_{*}\left(\bar{m}_{x}\right)=\bar{m}_{f(x)}
$$

pour tout $x \in \Sigma_{T}$, et telles que $\bar{m}_{x}=\psi(p, x)_{*}\left(\bar{m}_{p}\right)$ pour tout point $x$ dans $\left[0 ; a_{0}\right]$. En particulier, la mesure $\bar{m}_{p}$ est invariante par $A(p)$. Comme les valeurs propres de $B(p)$ ont multiplicité 1 et sont de modules distincts, $\bar{m}_{p}$ doit être supporté dans l'ensemble des directions propres $\left\{\xi_{j}: 1 \leqslant j \leqslant k\right\}$. De ce fait, $\bar{m}_{q}$ est supporté dans $\left\{\psi(p, q) \xi_{j}: 1 \leqslant j \leqslant\right.$ $k\}$ alors que la mesure $\bar{m}_{f^{l}(q)}$ est supporté, à la fois, dans $\left\{\psi\left(p, f^{l}(q)\right) \xi_{j}: 1 \leqslant j \leqslant k\right\}$ et dans $\left\{A^{l}(q) \psi(p, q) \xi_{j}: 1 \leqslant j \leqslant k\right\}$. Ceci n'est pas possible parce que, d'après la condition (8), les deux derniers ensembles sont disjoints. Nous sommes donc arrivés à une contradiction, ce qui démontre que les exposants de Lyapunov du cocycle $f_{A}$ pour $\mu$ ne sont pas tous nuls.

Théorème 7 reste vrai dans le cadre ses cocycles dominés réels :

Corollaire 1.19. - Pour chaque $C>0$ et $v \in] 0,1]$, il existe un sous-ensemble ouvert dense pour la topologie $C^{0}$ dans l'espace des applications $(C, v)$-Hölder continues $x \rightarrow A(x)$ à valeurs dans $S L(k, \mathbb{R})$ engendrant un cocycle dominé $f_{A}$ audessus d'un shift $f: \Sigma_{T} \rightarrow \Sigma_{T}$, telles que les exposants de Lyapunov du cocycle 
engendré ne sont pas tous nuls, pour toute mesure de probabilité $f$-invariante et $f$ ergodique avec la propriété de produit local.

Démonstration. - C'est une variation des arguments utilisés dans la preuve du Théorème 7. Nous pouvons supposer que $\Sigma_{T}$ contient plus qu'une orbite. Soit $p$ un point fixe (ou périodique) de $f$ et $q$ un point homocline associé à $p$, comme précédemment.

Nous considerons l'ensemble ouvert dense des applications $A$ telles que toutes les valeurs propres de $A(p)$ aient multiplicité 1 et, sauf pour les paires de valeurs propres complexes conjuguées, tous les modules soient distincts. Soient $\xi_{i}, 1 \leqslant i \leqslant r$ les points de $\mathbb{C} \mathbb{P}^{k-1}$ représentant les espaces propres des valeurs propres réelles, et $\eta_{j}, 1 \leqslant j \leqslant s$, les sous-espaces projectifs de dimension 1 représentant les espaces caractéristiques des valeurs propres complexes. Alors le support de toute mesure de probabilité invariante par $A(p)$ est contenue dans l'union $\left(\bigcup_{i} \xi_{i}\right) \cup\left(\bigcup_{j} \eta_{j}\right)$ de ces sous-espaces invariants.

Pour ces applications, nous demandons, de plus, que les images $A^{l}(q) \psi(p, q) \xi_{i}$, $A^{l}(q) \psi(p, q) \eta_{j}$ soient en position générale par rapport à $\psi\left(p, f^{l}(q)\right) \xi_{i}, \psi\left(p, f^{l}(q)\right) \eta_{j}$. Ceci défini un sous-ensemble ouvert d'applications $A: \Sigma_{T} \rightarrow S L(k, \mathbb{R})$, et on vérifie aisément que ce sous-ensemble est dense : on peut toujours perturber une application $A$ donnée, dans un voisinage de $f^{l-1}(q)$ disjoint du cylindre $\left[0 ; a_{0}\right]$ qui contient $p$, de façon que cette deuxième condition soit satisfaite.

Si la dimension de $\mathbb{C} P^{k-1}$ est au moins 3 , cela signifie que tous ces sous-espaces projectifs sont disjoints. De même si toutes les valeurs propres sont réelles. La démonstration est alors analogue à celle du Théorème 7 . Il nous reste à considérer deux cas seulement : $k=3$ et $A(p)$ a une valeur propre réelle et deux valeurs propres complexes, ou $k=2$ et $A(p)$ a deux valeurs propres complexes.

Considérons d'abord le cas $k=3$, avec une valeur propre réelle et deux valeurs propres complexes. Nous allons voir que pour ce sous-ensemble ouvert dense d'applications $A$, les exposants de Lyapunov du cocycle $f_{A}$ ne sont pas tous nuls. Comme $A^{l}(q) \psi(p, q) \eta_{1}$ et $\psi\left(p, f^{l}(q)\right) \eta_{1}$ sont en position générale, ils s'intersectent en un seul point $\tilde{\xi}$. Supposons qu'il existe une mesure de probabilité $m_{p}$ invariante par $A(p)$ et par les holonomies stable et instable. Alors son image par $\psi\left(p, f^{l}(q)\right)$ doit être supportée $\operatorname{par} \tilde{\xi}$, donc la mesure $m_{p}$ doit être une mesure de Dirac sur un point de $\eta_{1}$. Ceci n'est pas possible, car $A(p)$ n'a pas de point fixe sur $\eta_{1}$ : ses valeurs propres sont complexes non réelles.

Quand $k=2$ et que $A(p)$ a deux valeurs propres complexes, notre construction doit être légerement modifiée. Nous considérons l'ensemble dense (non-ouvert) des $A$ tels que les arguments des valeurs propres de $A(p)$ soient irrationnels. Alors, $A(p)$ a une seule mesure invariante $\lambda$ sur la fibre $\{p\} \times \mathbb{C P}^{1}$ de $p$, et c'est une mesure lisse. Comme les holonomies stable et instable sont des transformations projectives, $\lambda_{1}=A^{l-1}(q) \psi(p, q)_{*} \lambda$ et $\lambda_{2}=\psi\left(p, f^{l}(q)\right)_{*} \lambda$ sont aussi des mesures lisses sur les fibres de $f^{l-1}(q)$ et $f^{l}(q)$, respectivement.

Quitte à multiplier $A\left(f^{l-1}(q)\right)$ par une transformation hyperbolique (qui ne préserve aucune mesure lisse dans $\left.\mathbb{C P}^{1}\right)$ proche de l'identité, on peut supposer que $A\left(f^{l-1}(q)\right)$ n'envoie pas $\lambda_{1}$ sur $\lambda_{2}$. Ceci montre que l'ensemble des applications $A$ telles que les matrices $A(p)$ et $\psi\left(p, f^{l}(q)\right)^{-1} A^{l}(q) \psi(p, q)$ n'ont pas de mesure invariante commune est dense. 
D'autre part, l'ensemble des transformations dans la fibre de $p$ préservant une probabilité $A(p)$-invariante est fermé, parce que l'espace des probabilités dans la fibre est un compacte. Comme les holonomies varient continûment avec l'application $A$, d'après le Lemme 1.14, on en déduit que l'ensemble des cocycles pour lesquels les matrices $A(p)$ et $\psi\left(p, f^{l}(q)\right)^{-1} A^{l}(q) \psi(p, q)$ n'ont pas de mesure invariante commune est aussi ouvert.

Pour ce sous-ensemble ouvert dense d'applications $A$ que nous venons de construire, les exposants de Lyapunov du cocyle $f_{A}$ sont non-nuls. Ceci conclut.

\subsection{Mesure totale d'exposants non-nuls, dans les familles à paramètres}

Nous allons à présent voir que l'ensemble des cocycles ayant tous leurs exposants nuls est contenu dans un ensemble fermé qui est une union finie de sous-variétés de codimension positive ( 1 ou plus). Nous en déduisons que, pour un ouvert dense de familles à un paramètre $t \mapsto A_{t}$ à valeurs dans l'espace des cocycles, l'ensemble des valeurs de $t$ pour lesquels les exposants sont nuls est discret, donc de mesure de Lebesgue nulle. Ceci reste vrai pour des familles génériques à $l$ paramètres, $l \in \mathbb{N}$ : le phénomène "exposants de Lyapunov nuls" est de codimension infinie.

On considère l'espace $\left.\left.\mathcal{A}_{v}, v \in\right] 0,1\right]$, des applications $A: \Sigma_{T} \rightarrow S L(k, \mathbb{C})$ qui sont $v$-Hölder continues : c'est une sous-variété de l'espace de Banach des applications $v$ Hölder continues à valeurs dans l'espace vectoriel $\mathcal{M}(k, \mathbb{C})$, muni de la norme $v$-Hölder $\|A\|_{\nu}=\|A\|_{0}+H_{v}(A)$, où $\|A\|_{0}$ est la norme $C^{0}$ et $H_{v}(A)$ est la plus petite constante $C$ pour laquelle l'application $A$ est $(C, v)$-Hölder continue.

Proposition 1.20. - L'ensemble des applications $A \in \mathcal{A}_{v}$ dont les exposants de Lyapunov sont nuls, pour au moins une mesure de probabilité invariante ergodique avec structure de produit local, est contenu dans un sous-ensemble fermé qui est union finie de sous-variétés de codimension positive.

Démonstration. - Notons $\mathcal{N} \subset S L(k, \mathbb{C})$ l'ensemble des matrices de $S L(k, \mathbb{C})$ possédant au moins deux valeurs propres de même module. C'est un fermé qui est une union finie de sous-variétés de codimension positive. En remarquant que l'application qui à une famille $A$ associe sa valeur $A(p)$ en un point $p \in \Sigma_{T}$ est une submersion, on obtient que l'ensemble

$$
\mathcal{M}_{1}(p)=\{A \in \mathcal{A}: A(p) \in \mathcal{N}\}
$$

est un sous-ensemble fermé qui est une union finie de sous-variétés de codimension positive. L'union des sous-variétés de codimension au moins $s$ est aussi un fermé, pour tout entier $s \geqslant 1$.

On suppose à présent que $p$ est un point fixe (ou périodique) de $\Sigma_{T}$ et $q$ un point homocline associé au point $p$, tel qu'on l'a choisi dans la démonstration du Théorème 7 , dont nous gardons les notations.

Notons $\mathcal{A}_{v}(p)=\mathcal{A}_{v} \backslash \mathcal{M}_{1}(p)$. C'est un ouvert dense dans $\mathcal{A}_{v}$. Pour chaque $1 \leqslant j \leqslant k$ on considère l'application $\xi_{j}: \mathcal{A}_{v}(p) \rightarrow \mathbb{C P}^{k-1}$ qui à $A \in \mathcal{A}_{v}(p)$ associe la jème direction propre de la matrice $A(p)$. Ces applications sont différentiables, de classe $C^{\infty}$.

On considère aussi les applications d'holonomie instable $\phi^{u}=\phi^{u}(p, q): \mathcal{A}_{v} \rightarrow$ $S L(k, \mathbb{C})$ et stable $\phi^{s}=\phi^{s}\left(p, f^{l}(q)\right): \mathcal{A}_{v} \rightarrow S L(k, \mathbb{C})$, qui à un cocycle dominé $A$ 
associent ses holonomies le long des feuilletages instable et stable. Le lemme suivant sera démontré à la fin de la section :

LEMME 1.21. - Les applications $\phi^{s}$ et $\phi^{u}$ sont de classe $C^{1}$.

On en déduit que, pour tout $j$, l'application qui à $A \in \mathcal{A}_{v}(p)$ associe $\Phi_{j}(A)=$ $\left(\phi^{s}\right)^{-1} A^{l}(q) \phi^{u} \cdot \xi_{j}$ est de classe $C^{1}$. On vérifie aisément que, pour tout $i$ et $j$ l'application $\left(\Phi_{j}, \xi_{i}\right): \mathcal{A}_{v}(p) \rightarrow\left(\mathbb{C P}^{k-1}\right)^{2}$ est une submersion : pour cela, il suffit de remarquer que l'on peut perturber l'application $A^{l}(q)$ sans perturber $A(p)$ ni les holonomies $\phi^{s}$ et $\phi^{u}$. On en déduit que l'ensemble

$$
\mathcal{M}_{2}(p, q)=\left\{A \in \mathcal{A}_{v}(p): \text { il existe } i, j \text { tels que } \Phi_{i}(A)=\xi_{j}(A)\right\}
$$

est un fermé de $\mathcal{A}_{v}(p)$ qui est une union finie de sous-variétés de codimension $k-1 \geqslant 1$.

D'après le Théorème 6 , l'ensemble des applications telles que le cocycle engendr'e $f_{A}$ sont nuls est inclus dans le fermé $\mathcal{M}_{1}(p) \cup \mathcal{M}_{2}(p, q)$. Ceci conclut la démonstration de la Proposition 1.20.

COROLlaIRE 1.22. - Il existe un ouvert dense de familles à un paramètre $t \mapsto A_{t} \in$ $\mathcal{A}_{v}$ de classe $C^{r}, 1 \leqslant r \leqslant \infty$, pour lesquelles l'ensemble des valeur de t telles que les exposants de Lyapunov sont nuls, pour au moins une mesure de probabilité invariante ergodique avec structure de produit local, est discret, donc de mesure de Lebesgue zéro.

Démonstration. - On considère l'union des sous-variétés de codimension au moins 2 contenues dans $\mathcal{M}_{1}(p) \cap \mathcal{M}_{2}(p, q)$. C'est un fermé, et l'ensemble des familles à un paramètre qui ne le rencontrent pas forme un ouvert dense. Parmis ces familles, il reste à considérer celles qui sont transverses aux sous-variétés de codimension 1 contenues dans $\mathcal{M}_{1}(p) \cap \mathcal{M}_{2}(p, q)$; d'après la théorie de la transversalité, c'est encore un ouvert dense, ce qui conclut.

Remarque 1.23. - L'ensemble des applications $A \in \mathcal{A}_{v}$ pour lesquelles tous les exposants de Lyapunov sont nuls, pour une mesure de probabilité invariante ergodique avec structure de produit local, est en fait contenu dans l'intersection $\bigcap_{p}\left(\mathcal{M}_{1}(p) \cup\right.$ $\mathcal{M}_{2}(p, q)$ ), où $p$ parcours l'ensemble des points périodiques de $\Sigma_{T}$. Cet ensemble est contenu dans des fermés de codimension arbitrairement grande. Il s'en suit que le Corollaire 1.22 reste vrai pour les familles a $l$ paramètres, pour tout $l \in \mathbb{N}$.

\subsection{Démonstration du Lemme 1.21}

Démonstration. - La démonstration consiste à présenter explicitement l'expression des dérivées de ces applications. Nous traitons le cas de $\phi^{s}$, l'argument pour $\phi^{u}$ est analogue. Nous supposerons que $p$ est un point fixe, dans un cylindre $[0 ; a]$; le cas périodique $p \in\left[0 ; a_{0}, \ldots, a_{s-1}\right]$ est analogue : il suffit de remplacer $\theta_{a}^{i}$ par $\left(\theta_{a_{0}} \cdots \theta_{a_{s-1}}\right)^{[i / s]}$ dans les arguments.

Rappelons que $\phi^{s}$ est la limite uniforme des applications $\phi_{n}\left(p, f^{l}(q)\right)=A^{n}\left(f^{l}(q)\right)^{-1}$ $\times A^{n}(p)$. Notons $u=f^{l}(q)$, et $p_{i}=f^{i}(p)$ et $u_{i}=f^{l+i}(q)$ pour $i \geqslant 1$. On vérifie 
facilement que chaque application $\phi_{n}$ est de classe $C^{1}$, et un calcul directe donne

$$
\begin{gathered}
D \phi_{n}(A) H=\sum_{i=0}^{n-1} \alpha_{i, n}-\beta_{i, n} \\
\alpha_{i, n}=A^{i}(u)^{-1} \phi_{n-i}\left(p_{i}, u_{i}\right) A\left(p_{i}\right)^{-1} H\left(p_{i}\right) A^{i}(p) \quad \text { et } \\
\beta_{i, n}=A^{i}(u)^{-1} A\left(u_{i}\right)^{-1} H\left(u_{i}\right) \phi_{n-i}\left(p_{i}, u_{i}\right) A^{i}(p),
\end{gathered}
$$

pour point $A \in \mathcal{A}_{v}$ et tout vecteur tangent $H$. Soit

$$
\begin{gathered}
L(A) H=\sum_{i=0}^{\infty} \alpha_{i}-\beta_{i} \\
\alpha_{i}=A^{i}(u)^{-1} \phi\left(p_{i}, u_{i}\right) A\left(p_{i}\right)^{-1} H\left(p_{i}\right) A^{i}(p) \quad \text { et } \\
\beta_{i}=A^{i}(u)^{-1} A\left(u_{i}\right)^{-1} H\left(u_{i}\right) \phi\left(p_{i}, u_{i}\right) A^{i}(p) .
\end{gathered}
$$

Nous allons montrer que $L(A) H$ est bien définie (convergente) et que $L(A)$ est un opérateur linéaire borné. De plus, $D \phi_{n}(A)$ converge localement uniformément vers $L(A)$, par rapport à la norme des opérateurs linéaires. Nous en déduirons que $L(A)$ est la dérivée de $\phi^{s}$ à chaque point $A$.

Commençons par rappeler que, d'après la Remarque 1.13 , la norme de $\phi\left(p_{i}, u_{i}\right)$ id est bornée par $C_{4} d\left(p_{i}, u_{i}\right)^{\nu}$. Comme $u=f^{l}(q)$ est dans la variété stable locale de $p$, cette distance est bornée par $\theta_{a}^{i}$. Alors la condition de domination (6) implique que si on remplace $\phi\left(p_{i}, u_{i}\right)$ par l'identité dans $\alpha_{i}$, la norme n'en est affectée que par une constante additive bornée par $C_{0} C_{4} \hat{\tau}^{i}\left\|A^{-1}\right\|_{0}\|H\|_{0}$. La constante $C_{0}$ correspond à l'ordre $k$ à partir de laquelle (6) est valable; elle ne dépend que de la norme et d'un module de continuité de l'application $A$ et peut donc être choisie constante dans un voisinage de $A$. Dans la suite nous notons $C_{5}=C_{0} C_{4}\left\|A^{-1}\right\|_{0}$. De même, si l'on remplace $\phi\left(p_{i}, u_{i}\right)$ par l'identité dans $\beta_{i}$ la norme n'en est affectée que par une constante bornée par $C_{5} \hat{\tau}^{i}\|H\|_{0} \leqslant C_{5} \hat{\tau}^{i}\|H\|_{\nu}$.

Cela implique que la série dans (10) converge si et seulement si l'expression

$$
\sum_{i=0}^{\infty} A^{i}(u)^{-1} A\left(p_{i}\right)^{-1} H\left(p_{i}\right) A^{i}(p)-A^{i}(u)^{-1} A\left(u_{i}\right)^{-1} H\left(u_{i}\right) A^{i}(p)
$$

converge. Pour montrer que c'est bien le cas, remarquons que

$$
\left\|A\left(p_{i}\right)^{-1} H\left(p_{i}\right)-A\left(u_{i}\right)^{-1} H\left(u_{i}\right)\right\| \leqslant\left\|A^{-1}\right\|_{\nu}\|H\|_{\nu} d\left(p_{i}, u_{i}\right)^{\nu} \leqslant\left\|A^{-1}\right\|_{\nu}\|H\|_{\nu} \theta_{a}^{i \nu} .
$$

Si on combine cette borne avec la condition de domination (6), on obtient que le terme général de la série (11) est borné par $C_{6} \hat{\tau}^{i}\|H\|_{\nu}$, où $C_{6}=C_{0}\left\|A^{-1}\right\|_{\nu}$. Ceci montre que cette série converge, et définit bien un opérateur linéaire borné. Il en est alors de même pour la définition de $L(A)$. De plus, comme les constantes $C_{5}$ et $C_{6}$ sont bornées dans un voisinage de $A$, la convergence est localement uniforme. Il s'en suit que $L(A)$ dépend continûment de $A$. 
Montrons que $D \phi_{n}(A)$ converge uniformément vers $L(A)$ quand $n$ tend vers l'infini. D'après la Remarque 1.13 , pour tout $0 \leqslant i \leqslant n-1$,

$$
\left\|\phi_{n-i}\left(p_{i}, u_{i}\right)-\phi\left(p_{i}, u_{i}\right)\right\| \leqslant C_{4} \hat{\tau}^{n-i} d\left(p_{i}, u_{i}\right)^{v} \leqslant C_{4} \hat{\tau}^{n-i} \theta_{a}^{i v} .
$$

En utilisant la condition (6) une fois encore, on conclut que la différence $\alpha_{i, n}-\alpha_{i}$ est bornée par $C_{4} \hat{\tau}^{n-i} C_{0} \hat{\tau}^{i}\|H\|_{0}\left\|A^{-1}\right\|_{0} \leqslant C_{7} \hat{\tau}^{n}\|H\|_{v}$, où $C_{7}=C_{0} C_{4}\left\|A^{-1}\right\|_{0}$. La différence $\beta_{i, n}-\beta_{i}$ admet la même borne. D'autre part, nous avions démontré que la norme du terme général $\alpha_{i}-\beta_{i}$ de la série (10) est bornée par $\left(2 C_{5}+C_{6}\right) \hat{\tau}^{i}\|H\|_{\nu}$ pour tout $i \geqslant 0$. Ceci montre que

$$
\left\|D \phi_{n}(A) H-L(A) H\right\| \leqslant\left(\sum_{i=0}^{n-1} 2 C_{7} \hat{\tau}^{n}+\sum_{i=n}^{\infty}\left(2 C_{5}+C_{6}\right) \hat{\tau}^{i}\right)\|H\|_{\nu} .
$$

L'expression à droite converge vers zéro, uniformément dans un voisinage de $A$, quand $n$ tend vers l'infini. C'est à dire que $D \phi_{n}(A)$ converge vers $L(A)$ localement uniformément, en tant qu'opérateurs linéaires.

Nous avions montré que $\left(\phi_{n}\right)_{n}$ converge vers $\phi$, et nous venons de voir que la suite des dérivées $\left(D \phi_{n}\right)_{n}$ converge vers $L$. Dans les deux cas la convergence est localement uniforme. Il s'en suit que la limite $\phi$ est une application différentiable, et que sa dérivée coïncide avec $L$. Ceci conclut la démonstration du lemme.

Remarque 1.24. - La démonstration reste valable si l'on remplace la norme v-Hölder par une norme $\|\cdot\|$ plus fine (c'est à dire $\|\cdot\| \geqslant c\|\cdot\|_{\nu}$ ). Ceci nous permet d'appliquer le Lemme 1.21 dans le cadre des cocycles de classe $C^{r}$ que nous étudions à la Section 2.

\section{Cocycles au-dessus d'une dynamique hyperbolique}

Dans cette section, nous traduisons les principaux résultats de la Section 1 pour des cocycles au-dessus d'une dynamique hyperbolique.

\subsection{Cocycles $s$-dominés : cadre géométrique}

Dans la suite on appellera cocycle projectif mesuré la donnée de $(M, f, \mu, \mathcal{E}, F, m)$ vérifiant

1. $f: M \rightarrow M$ est un difféomorphisme $C^{1}$ d'une variété Riemanniene compacte $M$, et $\Lambda$ est un ensemble basique hyperbolique de $f$;

2. $\mu$ est l'état d'équilibre d'un potentiel Hölder continu à support dans $\Lambda$;

3. $\pi: \mathcal{E} \rightarrow M$ est un fibré projectif sur $M$ et $F: \mathcal{E} \rightarrow \mathcal{E}$ est un cocycle projectif $C^{1}$ au-dessus de $f$;

4. $m$ est une mesure de probabilité borelienne $F$-invariante dans $\hat{\Lambda}=\pi^{-1}(\Lambda)$ telle que $\pi_{*}(m)=\mu$.

Comme précédemment on note $\left(m_{x}\right)_{x \in M}$ une désintégration de la mesure $m$ le long des fibres $\mathcal{E}_{x}=\pi^{-1}(x)$.

Remarque 2.1. - Dans la définition ci-dessus, il suffit de supposer que $\mu$ est une mesure borélienne de probabilité, $f$-invariante ergodique, dont le support coïncide avec 
$\Lambda$, et dont le relevé sur les sous-shifts de type fini, via une partition de Markov (voir ci-dessous), possède la propriété de produit local. C'est le cas pour tout état d'équilibre d'un potentiel Hölder continu, voir [2,12,19].

On notera $T_{\Lambda} M=E^{u} \oplus E^{s}$ la décomposition invariante correspondant à l'ensemble hyperbolique $\Lambda$ de $f$. Le fibré dilatant $E^{u}$ sera aussi appelé direction instable, alors que le fibré contractant $E^{s}$ sera appelé direction stable. On suppose que la variété $\mathcal{E}$ est aussi munie d'une metrique Riemanniene.

DÉFINITION 2.2. - On dit que le cocycle $F: \mathcal{E} \rightarrow \mathcal{E}$ est dominé dans la direction stable, ou s-dominé, au-dessus de $\Lambda$ si l'ensemble $\hat{\Lambda}$ est partiellement hyperbolique pour $F$ avec décomposition invariante $T_{\hat{\Lambda}} \mathcal{E}=E^{c u} \oplus E^{s s}$ telle que

$$
E^{c u}=D \pi^{-1}\left(E^{u}\right) \quad \text { et } \quad D \pi\left(E^{s s}\right)=E^{s} .
$$

On définit de même un cocycle dominé dans la direction instable, ou u-dominé, audessus de $\Lambda$.

D'après la théorie de l'hyperbolicité normale, [9,13], si $F$ est $s$-dominé, il admet un seul feuilletage invariant $\mathcal{F}^{s s}$ partout tangent au fibré stable $E^{s s}$. Les feuilles de ce feuilletage stable-fort $\mathcal{F}^{s s}$ sont des sous-variétés $C^{1}$ immergées dans $\mathcal{E}$ et elles se projettent sur les variétés stables des points de $\Lambda$. Le but de ce paragraphe est de démontrer le

ThÉORÈme 8. - Soit $(M, f, \mu, \mathcal{E}, F, m)$ un cocycle projectif mesuré tel que le cocycle $F$ est $s$-dominé et tous ses exposants de Lyapunov pour $\mu$ sont nuls.

Alors les mesures conditionelles $\left(m_{x}\right)_{x}$ de $m$ sont $\mu$-presque partout preservées par l'holonomie du feuilletage stable-fort : il existe un ensemble $E \subset \Lambda$ avec $\mu(E)=1$ tel que, pour $x, y \in E$ dans une même variété stable de $f$, la projection de $\mathcal{E}_{x}$ à $\mathcal{E}_{y}$ le long des feuilles de $\mathcal{F}^{s s}$ envoie $m_{x}$ sur $m_{y}$.

La démonstration de ce résultat sera donnée à la fin du paragraphe.

Le premier lemme est une traduction de la notion de $s$-domination, afin de la rendre compatible avec la notion de $s$-domination de la Section 1. On note $F_{x}: \mathcal{E}_{x} \rightarrow \mathcal{E}_{f(x)}$ l'action (projective) de $F$ dans la fibre d'un point $x$. On note $\left\|F_{x}\right\|$ la norme de n'importe quelle réalisation de $F_{x}$ par une application linéaire de déterminant 1 (voir les commentaires précédant le Théorème 1$)$.

Lemme 2.3. - Le cocycle $F: \mathcal{E} \rightarrow \mathcal{E}$ est $s$-dominé au-dessus de $\Lambda$ si et seulement si il existe $L \in \mathbb{N}$ et $\tau<1$ tels que, pour tout $x \in \Lambda$,

$$
\left\|F_{x}^{L}\right\|\left\|\left(F_{x}^{L}\right)^{-1}\right\|\left\|D f^{L} \mid E_{x}^{s}\right\| \leqslant \tau .
$$

Démonstration. - Supposons que le cocycle $F$ est dominé. Alors, d'après les définitions, il existe $N \geqslant 1$ et une décomposition invariante $T_{\tilde{\Lambda}} M=E^{c u} \oplus E^{s s}$ telle que $E^{c u}=D \pi^{-1}\left(E^{u}\right), E^{s s}$ se projette sur la direction stable $E^{s}$ de $f$, et on a

$$
\sup _{z \in \hat{\Lambda}}\left\|D F^{N} \mid E_{z}^{s s}\right\|\left\|\left(D F^{N} \mid E_{z}^{c u}\right)^{-1}\right\| \leqslant \frac{1}{2} .
$$


Remarquons qu'il existe une constante $C_{1}>0$, qui ne dépend que de $F$ et du choix de $E^{s s}$, telle que

$$
\left\|D f^{j N}\left|E_{\pi(z)}^{s}\left\|\leqslant C_{1}\right\| D F^{j N}\right| E_{z}^{s s}\right\|
$$

pour tout $j \geqslant 1$ et tout $z \in \mathcal{E}$. En effet, ces deux transformations linéaires sont conjuguées par la dérivée de la projection qui envoie $E_{z}^{s s}$ isomorphiquement sur $E_{\pi(z)}^{s}$. D'autre part, $E^{c u}$ contient le fibré $E^{c}=D \pi^{-1}(0)$ tangent aux fibres de $\mathcal{E}$. En particulier,

$$
\left\|\left(D F^{l} \mid E_{z}^{c}\right)^{-1}\right\| \leqslant\left\|\left(D F^{l} \mid E_{z}^{c u}\right)^{-1}\right\|
$$

à tout point $z \in \mathcal{E}$ et pour tout $l \geqslant 1$. De plus, comme la restriction de $F^{l}$ à chaque fibre $\mathcal{E}_{x}=\pi^{-1}(x)$ est le projectivisé d'une application linéaire $\hat{F}_{x}^{l}$ et que, par définition, $\left\|F_{x}^{l}\right\|=\left\|\hat{F}_{x}^{l}\right\|$,

$$
\sup _{z \in \mathcal{E}_{x}}\left\|\left(D F^{l} \mid E_{z}^{c}\right)^{-1}\right\|=\left\|F_{x}^{l}\right\|\left\|\left(F_{x}^{l}\right)^{-1}\right\|
$$

pour tout $x \in M$ et $l \geqslant 1$.

Les relations que nous venons d'obtenir entrainent que

$$
\left\|F_{x}^{j N}\right\|\left\|\left(F_{x}^{j N}\right)^{-1}\right\|^{\prime}\left\|D f^{j N}\left|E_{x}^{s}\left\|\leqslant \sup _{z \in \mathcal{E}_{x}}\right\|\left(D F^{j N} \mid E_{z}^{c u}\right)^{-1}\left\|C_{1}\right\| D F^{j N}\right| E_{z}^{s s}\right\| \leqslant C_{1} 2^{-j}
$$

pour tout $j \geqslant 1$ et tout $x \in M$. Maintenant, pour avoir la relation (12) il suffit de fixer $j \geqslant 1$ suffisament grand pour que $C_{1} 2^{-j}$ soit plus petit que 1 , et de prendre $\tau=C_{1} 2^{-j}$ et $L=j N$.

La réciproque, c'est à dire, le fait que (12) implique que le cocycle est $s$-dominé, se démontre de façon similaire. Comme nous n'aurons pas l'occasion de l'utiliser dans cet article, nous laissons la démonstration pour le lecteur.

Remarquons que l'énoncé du Théorème 8 ne change pas si on remplace $f$ par un itéré positif : le feuilletage stable-fort de $f$ l'est aussi pour tous les $f^{j}$ avec $j \geqslant 1$. Nous pouvons donc supposer que $N=L=1$ dans les relations (1), (2) et (12), et nous le ferons dans la suite.

Nous allons utiliser le fait suivant, dont la démonstration peut être trouvée dans [7] : pour tout ensemble basique hyperbolique $\Lambda$ et tout $\varepsilon>0$ il existe un sous-shift transitif de type fini $\sigma: \Sigma_{T} \rightarrow \Sigma_{T}$ et une application continue surjective $P: \Sigma_{T} \rightarrow \Lambda$ telle que

1. $P$ est une semi-conjugaison entre $f$ et $\sigma$, c'est à dire, $P \circ \sigma=f \circ P$;

2. P est injective au-dessus d'un sous-ensemble $G_{\delta}$ dense de $\Lambda$;

3. ce sous-ensemble $G_{\delta}$ dense est de mesure $\mu$ totale;

4. le diamètre de tous les rectangles de Markov $R_{i}=P([0 ; i])$ est borné par $\varepsilon$.

On appellera partition de Markov à la fois l'application $P$ et la famille des rectangles $R_{i}$.

Remarquons que si $\varepsilon>0$ est choisi suffisament petit, en utilisant le Lemme 2.3 et la continuité de $y \mapsto\left\|D F \mid E_{y}^{s}\right\|$ on peut trouver $\tau<\hat{\tau}<1$ et des majorants $\theta_{i}$ pour $\left\|D F \mid E_{y}^{s}\right\|$ dans le voisinage de rayon $\varepsilon$ autour de chaque $R_{i}$, tels que

$$
\left\|F_{x}\right\|\left\|\left(F_{x}\right)^{-1}\right\| \theta_{i}<\hat{\tau}
$$


pour tout $x \in R_{i}$ et $i \in\{1, \ldots, n\}$. Dans la suite nous supposerons qu'une partition de Markov a été fixée vérifiant (13).

La propriété 3 de la partition de Markov nous permet de relever $\mu$ en une probabilité $\sigma$-invariante $\tilde{\mu}$ dans $\Sigma_{T}$, qui est aussi ergodique. Dans quelques instants nous expliquerons comment on peut aussi associer au fibré $\mathcal{E}$, au cocycle $F$ et à la mesure $F$ invariante $m$ des objets correspondants au-dessus de $\Sigma_{T}, \sigma$ et $\tilde{\mu}$.

LEMME 2.4. - Avec les notations ci-dessus, il existe une distance $d_{\Sigma}$ sur le sous-shift $\Sigma_{T}$, dans la classe Hölder des distances $d_{\theta}$, telle que

- $d_{\Sigma}(\sigma(p), \sigma(q)) \leqslant \theta_{i} d_{\Sigma}(p, q)$ pour tout $p, q \in[0 ; i]$ tels que $q \in W_{\mathrm{loc}}^{s}(p)$;

- $P$ est Lipschitz continue par rapport à la distance $d_{\Sigma}$.

Démonstration. - Pour $p=\left(p_{j}\right)_{j}$ et $q=\left(q_{j}\right)_{j}$ dans un cylindre [0;i], définissons $D_{u}(p, q)$ et $D_{s}(p, q)$ comme étant les plus grands entiers (non-négatifs, eventuellement infinis) tels que

$$
p_{j}=q_{j} \quad \text { pour tout }-D_{s}(p, q) \leqslant j \leqslant D_{u}(p, q) .
$$

Soient $\theta_{1}, \ldots, \theta_{n}<1$ les constantes dans (13) et fixons aussi un majorant $\theta_{0}<1$ quelconque pour $\left\|\left(D f \mid E^{u}\right)^{-1}\right\|$. Alors, définissons

$$
\begin{aligned}
& d^{u}(p, q)=\theta_{0}^{D_{u}(p, q)}, \quad d^{s}(p, q)=\prod_{j=1}^{D_{s}(p, q)} \theta_{p_{-j}}, \quad \text { et } \\
& d_{\Sigma}(p, q)=\max \left\{d^{u}(p, q), d^{s}(p, q)\right\} .
\end{aligned}
$$

Si $p$ et $q$ ne sont pas dans un même cylindre, on prend simplement $d_{\Sigma}(p, q)=$ $d_{s}(p, q)=d_{u}(p, q)=1$.

Remarquons que $d^{u}(p, q)=0$ si et seulement si $D_{u}(p, q)=\infty$, c'est à dire, si et seulement si $p$ et $q$ sont dans la même variété stable locale. De même pour $d^{s}(p, q)$, $D_{s}(p, q)$ et la variété instable locale. En particulier, $d_{\Sigma}(p, q)=0$ si et seulement si $p=q$. Il est aussi facile de voir que $d_{\Sigma}(p, q)=d_{\Sigma}(q, p)$. Finalement, $D_{*}(p, r) \geqslant$ $\min \left\{D_{*}(p, q), D_{*}(q, r)\right\}$ pour tout $p, q, r$ et $*=u$ et $*=s$. En conséquence, $d_{*}(p, r) \leqslant$ $\max \left\{d_{*}(p, q), d_{*}(q, r)\right\}$ pour $*=u$ et $*=s$, et donc

$$
d_{\Sigma}(p, q) \leqslant \max \left\{d_{\Sigma}(p, q), d_{\Sigma}(q, r)\right\} .
$$

Ceci montre que $d_{\Sigma}$ est une métrique (il s'agit même d'une ultra-métrique).

Vérifions à présent les propriétés annoncées. Fixons $\theta \in] 0,1[$ et $v \in] 0,1]$ tels que $\theta^{1 / v} \leqslant \theta_{i} \leqslant \theta^{v}$, pour tout $i$. On vérifie aisément que l'identité est un homéomorphisme Hölder continu, d'inverse continu, entre $\left(\Sigma_{T}, d_{\theta}\right)$ et $\left(\Sigma_{T}, d_{\Sigma}\right)$. Donc, la distance $d_{\Sigma}$ est dans la classe Hölder des distances $d_{\theta}$.

Comme on l'a remarqué auparavant, si $q$ appartient à la variété stable locale de $p$ on a $D_{u}(p, q)=\infty$ et $d_{u}(p, q)=0$. De plus, $\sigma(p)$ et $\sigma(q)$ sont aussi dans une même variété stable locale, et $D_{s}(\sigma(p), \sigma(q))=D_{s}(p, q)+1$. Ceci donne la première propriété :

$$
d_{\Sigma}(\sigma(p), \sigma(q))=d_{s}(\sigma(p), \sigma(q))=\prod_{j=0}^{D_{s}(p, q)} \theta_{p_{-j}}=\theta_{p_{0}} \prod_{j=1}^{D_{s}(p, q)} \theta_{p_{-j}}
$$




$$
=\theta_{p_{0}} d_{s}(p, q)=\theta_{p_{0}} d_{\Sigma}(p, q) .
$$

Pour avoir la deuxième propriété, il suffit de montrer que la restriction de $P$ à chaque variété stable locale et instable locale est Lipschitz avec constante de Lipschitz uniforme $C_{0}$. En effet, supposons que ceci est connu. Pour toute paire de points $p$ et $q$ dans un même cylindre $[0 ; i]$ considérons le point $r$ d'intersection entre la variété stable locale de $p$ et la variété instable locale de $q$. Alors,

$d(P(p), P(q)) \leqslant C_{0} d_{\Sigma}(p, r)+C_{0} d_{\Sigma}(r, q)=C_{0} d_{s}(p, q)+C_{0} d_{u}(p, q) \leqslant 2 C_{0} d_{\Sigma}(p, q)$.

Ceci donne la propriété de Lipschitz pour des points dans un même cylindre. D'autre part, si $p$ et $q$ ne sont pas dans un même cylindre, on a $d_{\Sigma}(p, q)=1$ et la propriété de Lipschitz est automatique : il suffit qu'on prenne la constante de Lipschitz plus grande que le diamètre de l'image $\Lambda$ de l'application $P$. Nous avons donc bien réduit la démonstration du lemme à montrer que $P$ est uniformément Lipschitz continue sur les variétés stables locales et instables locales.

Soit $p, q \in \Sigma_{m}$ tels que $q \in W_{\mathrm{loc}}^{s}(q)$, et notons $x=P(p)$ et $y=P(q)$. Le choix de $P$ implique que la distance entre $f^{j}(x)=P\left(\sigma^{j}(x)\right)$ et $f^{j}(y)=P\left(\sigma^{j}(y)\right)$ est borné par $\varepsilon$ pour tout $j \geqslant 0$. C'est à dire, $y$ appartient à la $\varepsilon$-variété stable $W_{\varepsilon}^{s}(x)$ de $x$. Pour les mêmes raisons, on a que $f^{-j}(y)$ est dans $W_{\varepsilon}^{s}\left(f^{-j}(x)\right)$ pour tout $1 \leqslant j \leqslant D_{s}(p, q)$. D'autre part, notre choix des constantes $\theta_{i}$ implique que

$$
d\left(f^{-j+1}(x), f^{-j+1}(y)\right) \leqslant \theta_{p_{-j}} d\left(f^{-j}(x), f^{-j}(y)\right)
$$

pour tout $1 \leqslant j \leqslant D_{s}(p, q)$. En conséquence

$$
d(x, y) \leqslant\left(\prod_{j=1}^{D_{s}(p, q)} \theta_{p_{-j}}\right) d\left(f^{-D_{s}(p, q)}(x), f^{-D_{s}(p, q)}(y)\right) \leqslant d_{\Sigma}(p, q) \varepsilon .
$$

(Pour être complètement rigoureux, on devrait considérer des distances le long des variétés stables (longueurs des courbes les plus courtes joignant deux points dans la sous-variété) au lieu de la distance $d$ dans l'ambiant $M$. Cependant, les $\varepsilon$ variétés stables locales forment une famille continue de disques petits $C^{1}$ plongés dans $M$. Les deux sortes de distances ne diffèrent donc que d'un facteur proche de 1 , ce qui veut dire que l'estimé final (14) ne change que par un facteur aussi proche de 1 que l'on veut.)

La relation (14) montre que $P$ est bien Lipschitz sur les variétés stables locales. La démonstration pour les variétés instables locales est analogue, en utilisant le fait qu'on a choisit $\theta_{0}<1$ majorant le taux de contraction de $f^{-1}$ dans la direction $E^{u}$.

Remarque 2.5. - Si le cocycle est simultanément $s$-dominé et $u$-dominé, on peut construire $d_{\Sigma}$ de façon que la propriété de contraction donnée par le Lemme 2.4 soit valable simultanément dans les directions stable et instable.

Plus précisement, comme on l'avait fait pour (13), on peut choisir des majorants $\theta_{i}^{s}<1$ et $\theta_{i}^{u}<1$ pour, respectivement, $\left\|D f \mid E^{s}\right\|$ et $\left\|D f \mid E^{u}\right\|$ dans chaque rectangle $R_{i}$, tels que

$$
\left\|F_{x}\right\|\left\|\left(F_{x}\right)^{-1}\right\| \theta_{i}^{s}<\hat{\tau}<1 \quad \text { et } \quad\left\|\left(F_{x}\right)^{-1}\right\|\left\|F_{x}\right\| \theta_{i}^{u}<\hat{\tau}<1
$$


pour tout $x \in[0 ; i]$ et tout symbole $i$. Alors, en remplaçant la distance $d_{u}$ dans la démonstration du Lemme 2.4 par

$$
d_{u}(p, q)=\prod_{j=1}^{D_{u}(p, q)} \theta_{p_{j}},
$$

on obtient une distance $d_{\Sigma}$ telle que $P$ soit Lipschitz et qu'on ait les deux relations de contraction

$$
d_{\Sigma}(\sigma(p), \sigma(q)) \leqslant \theta_{i}^{s} d_{\Sigma}(p, q) \quad \text { et } \quad d_{\Sigma}\left(\sigma^{-1}(p), \sigma^{-1}(r)\right) \leqslant \theta_{i}^{u} d_{\Sigma}(p, r)
$$

pour tout $p \in \Sigma_{T}, q \in W_{\mathrm{loc}}^{s}(p)$ et $r \in W_{\mathrm{loc}}^{u}(p)$.

Notons $\tilde{\mathcal{E}}$ le fibré projectif au dessus de $\Sigma_{T}$ obtenu comme pull-back par $P$ du fibré $\mathcal{E}$ aud dessus de $\Lambda$ : rapplelons que $\tilde{\mathcal{E}}$ est l'ensemble des $(p, e) \in \Sigma_{T} \times \mathcal{E}$ tels que $P(p)=\pi(e)$. La fibre $\tilde{\mathcal{E}}_{p}$ est canoniquement identifiée à la fibre $\mathcal{E}_{x}$ où $x=P(p)$.

Ceci permet de relever le cocycle $F$ de façon canonique en un cocycle $\tilde{F}: \tilde{\mathcal{E}} \rightarrow \tilde{\mathcal{E}}$ audessus du sous-shift $\Sigma_{T}$ : on définit $\tilde{F}_{p}: \tilde{\mathcal{E}}_{p} \rightarrow \tilde{\mathcal{E}}_{\sigma(p)}$ comme étant la même application que $F_{x}: \mathcal{E}_{x} \rightarrow \mathcal{E}_{f(x)}, x=P(p)$, modulo l'identification des fibres $\tilde{\mathcal{E}}_{p}$ et $\tilde{\mathcal{E}}_{\sigma(p)}$ avec les fibres $\mathcal{E}_{x}$ et $\mathcal{E}_{f(x)}$. Ceci a un sens parce que $P(\sigma(p))=f(x)$.

L'application $\tilde{F}$ ainsi défini est clairement continue. Comme le fibré $\mathcal{E}$ est localement trivial, le fibré $\tilde{\mathcal{E}}$ est lui aussi localement trivial. Comme $\Sigma_{T}$ est totalement discontinu, on peut choisir de façon continue des bases orthonormées dans les fibres, ce qui donne une trivialisation de $\tilde{\mathcal{E}}$ qui induit une isométrie dans chaque fibre. On écrira donc $\tilde{\mathcal{E}}=\Sigma_{T} \times \mathbb{C P}^{k-1}$. On peut donc trouver une application continue $A: \Sigma_{T} \rightarrow S L(k, \mathbb{C})$ telle que $\tilde{F}$ coïncide avec le cocycle $f_{A}$ engendré par $A$. En particulier, les fonctions $\log \|A\|$ et $\log \left\|A^{-1}\right\|$ sont $\tilde{\mu}$-intégrables.

Finalement, le fait que le lieu d'injectivité de $P$ soit de $\mu$-mesure totale implique que l'on peut aussi relever $m$ en une mesure de probabilité $\tilde{m}$ dans $\tilde{\mathcal{E}}$ se projettant sur $\tilde{\mu}$ et dont les mesures conditionnelles sont données par $\tilde{m}_{p}=m_{x}$ avec $x=P(p)$, via l'identification de $\tilde{\mathcal{E}}_{p}$ et de $\mathcal{E}_{x}$.

Ceci nous ramène dans le cadre de la Section 1 : nous avons expliqué comment, à tout cocycle projectif mesuré $(M, f, \mu, \mathcal{E}, F, m)$ au-dessus d'une dynamique hyperbolique, on peut associer un cocycle projectif mesuré $\left(\Sigma_{T}, \sigma, \tilde{\mu}, A, \tilde{F}, \tilde{m}\right)$ au-dessus d'un sousshift de type fini. Il est facile de voir que les exposants de Lyapunov de $\tilde{F}$ pour $\tilde{\mu}$ et de $F$ pour $\mu$ sont les mêmes. Dans la suite, nous supposerons que ces exposants sont tous nuls, et utiliserons les résultats de la Section 1.4 pour en déduire le Théorème 8.

Nous avons besoin de savoir que le relèvement préserve la propriété de $s$-domination :

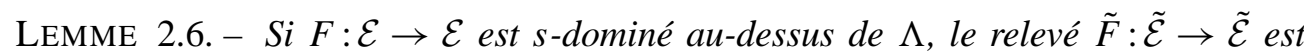
s-dominé.

Démonstration. - Prennons $d=d_{\Sigma}, v=1, N=1$, et des constantes $\hat{\tau}, \theta_{1}, \ldots, \theta_{n}<1$ satisfaisant l'inégalité (13). La première condition dans la Définition 1.11 est donnée par le Lemme 2.4. De la même façon, le fait que $P$ soit Lipschitz par rapport à la métrique $d=d_{\Sigma}$ implique que l'application $p \mapsto \tilde{F}_{p}$ l'est aussi : en effet le cocycle $F$ 
a été pris différentiable, donc Lipschitz et, comme on l'a expliqué précédemment, le relevé est donné, simplement, par $A(p)=\tilde{F}_{p}=F_{x}$ où $x=P(p)$. Ceci donne la deuxième condition. Finalement, en remarquant que $\|A(p)\|=\left\|F_{x}\right\|$, la troisième condition, $\|A(p)\|\left\|A(p)^{-1}\right\| \theta_{i}<\hat{\tau}$, dans la définition est une conséquence directe de l'inégalité (13).

Nous pouvons donc appliquer le Lemme 1.12 et la Proposition 1.16 au cocycle projectif mesuré $\left(\Sigma_{T}, \sigma, \tilde{\mu}, A, \tilde{F}, \tilde{m}\right)$ pour obtenir une famille de transformations inversibles $\phi(p, q): \mathbb{C} \mathbb{P}^{k-1} \rightarrow \mathbb{C} \mathbb{P}^{k-1}$, définies pour tout $p$ et $q$ dans une même variété stable locale, qui préservent les mesures conditionelles de $\tilde{m}$ :

$$
\phi(p, q)_{*} \tilde{m}_{p}=\tilde{m}_{q}
$$

pour tout $p$ et $q$ dans un sous-ensemble $\tilde{E}$ de $\tilde{\mu}$-mesure totale de $\Sigma_{T}$. Nous allons montrer que ces applications correspondent aux transformations d'holonomie locale (projection le long des feuilles) du feuilletage stable-fort du cocycle $F: \mathcal{E} \rightarrow \mathcal{E}$.

LEMME 2.7. - Soit $p$ et $q$ deux points dans une même variété stable locale dans $\Sigma_{T}$, et soit $x=P(p)$ et $y=P(q)$. Soit $h(x, y): \mathcal{E}_{x} \rightarrow \mathcal{E}_{y}$ la transformation d'holonomie locale associé au feuilletage stable-fort du cocycle $F$.

Alors, $\phi(p, q)=h(x, y)$.

Démonstration. - Le feuilletage stable-fort est caractérisé par le fait que l'image $\eta=$ $h(x, y) \xi$ d'un point quelconque $\xi \in \mathcal{E}_{x}=\tilde{\mathcal{E}}_{p}=\mathbb{C P}^{k-1}$ par l'holonomie du feuilletage est le seul point $\eta$ de la fibre $\mathcal{E}_{y}=\tilde{\mathcal{E}}_{q}$ dont l'orbite se rapproche de celle de $\xi$ strictement plus vite que la contraction maximale obtenu dans la direction centrale : il existe $C>0$ et $\alpha<1$ tel que

$$
d\left(F_{x}^{n}(\xi), F_{y}^{n}(\eta)\right) \leqslant C \alpha^{n}\left\|\left(F_{y}^{n}\right)^{-1}\right\|^{-1}\left\|F_{y}^{n}\right\|^{-1}
$$

pour tout $n \geqslant 1$. Il nous suffit donc de montrer que (16) est vraie pour $\eta=\phi(x, y) \xi$.

Pour cela, commençons par rappeler que, d'après la deuxième partie du Lemme 1.12,

$$
F_{y}^{n} \phi(p, q) \xi=\phi\left(\sigma^{n}(p), \sigma^{n}(q)\right) F_{x}^{n} \xi
$$

En utilisant la Remarque 1.13 pour $l=0$ et $v=1$, on en déduit que

$$
d\left(F_{x}^{n} \xi, F_{y}^{n} \phi(p, q) \xi\right) \leqslant C d_{\Sigma}\left(\sigma^{n}(p), \sigma^{n}(q)\right)
$$

où la constante $C>0$ ne dépend ni de $n$ ni des points $p$ et $q$. Comme $p$ et $q$ ont été pris dans une même variété stable locale, Lemme 2.4 implique

$$
d_{\Sigma}\left(\sigma^{n}(p), \sigma^{n}(q)\right) \leqslant \prod_{j=0}^{n-1} \theta_{p_{j}} .
$$


Rappelons aussi que les constantes $\theta_{i}$ ont été choisies satisfaisant (13) : c'est à dire, $\left\|F_{z}\right\|\left\|F_{z}^{-1}\right\| \theta_{i}<\hat{\tau}$ pour tout $z$ dans chaque cylindre $[0 ; i]$. Ces inégalités entrainent

$$
d\left(F_{x}^{n} \xi, F_{y}^{n} \phi(p, q) \xi\right) \leqslant C \prod_{j=0}^{n-1} \hat{\tau}\left\|F_{f^{j}(y)}\right\|^{-1}\left\|F_{f^{j}(y)}^{-1}\right\|^{-1} \leqslant C \hat{\tau}^{n}\left\|F_{y}^{n}\right\|^{-1}\left\|\left(F_{y}^{n}\right)^{-1}\right\|^{-1} .
$$

Il suffit donc de prendre $\alpha=\hat{\tau}$.

Remarque 2.8. - Voici deux conséquences immediates du Lemme 2.7 (qui peuvent être démontrées aussi directement) :

1. L'holonomie du feuilletage stable-fort du cocycle $F$ est projective.

2. Si $P(p)=P\left(p^{\prime}\right)$ et $P(q)=P\left(q^{\prime}\right)$ avec $p \in W_{\mathrm{loc}}^{s}(p)$ et $q^{\prime} \in W_{\mathrm{loc}}^{s}\left(p^{\prime}\right)$, alors $\phi(p, q)=\phi\left(p^{\prime}, q^{\prime}\right)$.

Maintenant nous pouvons compléter la démonstration du Théorème 8 :

Démonstration. - Rappelons qu'on considère la désintegration $\left(\tilde{m}_{p}\right)_{p}$ de la mesure $\tilde{m}$ décrite $\operatorname{par} \tilde{m}_{p}=m_{P(p)}$ pour tout $p$. D'après la Proposition 1.16, il existe un ensemble $\tilde{E}$ de $\tilde{\mu}$-mesure totale tel que $\phi(p, q)_{*} \tilde{m}_{p}=\tilde{m}_{q}$ pour tout $p$ et $q$ dans $\tilde{E}$. Notons $U$ un sousensemble $G_{\delta}$ dense de $\Lambda$ de $\mu$-mesure totale au-dessus duquel la partition de Markov est injective. Soit $R$ l'ensemble des points réguliers de $\mu$ (qui est aussi de $\mu$-mesure totale, car $\mu$ est ergodique). Notons encore $I$ l'ensemble des points $x \in \Lambda$ tels que $m_{f(x)}=\left(F_{x}\right)_{*} m_{x}$ : comme $m$ est invariante et que $f$ est inversible, il s'agit encore d'un ensemble de $\mu$-mesure totale. Soit $E_{0}$ un sous-ensemble mesurable de $\mu$-mesure totale contenu dans $U \cap R \cap I$ et dont la pré-image $P^{-1}\left(E_{0}\right)$ est contenue dans $\tilde{E}$. Apellons $E$ l'intersection de tous les itérés $f^{n}\left(E_{0}\right)$ avec $n \in \mathbb{Z}$. C'est encore un ensemble de $\mu$ mesure totale qui est, cette fois, invariant par $f$. Nous allons montrer que $E$ satisfait la conclusion du théorème.

Étant donnés deux points $x, y \in E$ dans une même variété stable on peut trouver $n \geqslant 1$ tel que $f^{n}(x)$ et $f^{n}(y)$ soient dans l'intérieur d'un même rectangle $R_{i}$. En effet, comme $x$ est un point générique pour $\mu$ et que $\mu$ charge tout ouvert, l'orbite future de $x$ passe un nombre infini de fois à une distance des bords des rectangles plus grande qu'un certain $\delta$ fixé. D'autre part la distance entre les itérés de $x$ et $y$ tend vers zero quand le temps tend vers l'infini. D'après (15) et le Lemme 2.7, on a que $h\left(f^{n}(x), f^{n}(y)\right)_{*} m_{f^{n}(x)}=m_{f^{n}(y)}$. L'invariance du feuilletage stable-fort implique alors $h\left(f^{n}(x), f^{n}(y)\right) \circ F_{x}^{n}=F_{y}^{n} \circ h(x, y)$. Comme tous les itérés de $x$ et $y$ sont contenus dans $I$, ceci implique que $h(x, y)_{*} m_{x}=m_{y}$.

\subsection{Cocycles dominées : cadre géométrique}

Nous conservons les notations de la section précédente.

DÉFINITION 2.9. - On dit que le cocycle $F: N \rightarrow N$ est dominé au-dessus de $\Lambda$ si l'ensemble $\hat{\Lambda}$ est fortement partiellement hyperbolique pour $F$ avec décomposition $T_{\hat{\Lambda}} N=E^{u u} \oplus E^{c} \oplus E^{s s}$ telle que

$$
D \pi\left(E^{u u}\right)=E^{u}, \quad E^{c}=D \pi^{-1}(0) \quad \text { et } \quad D \pi\left(E^{s s}\right)=E^{s} .
$$


D'après la théorie de l'hyperbolicité normale, [9,13], il existe alors deux feuilletages invariants $\mathcal{F}^{u u}$ et $\mathcal{F}^{s s}$ partout tangents aux fibrés $E^{u u}$ et $E^{s s}$, respectivement. Les feuilles se projettent, respectivement, sur les variétés instables et stables des points de $\Lambda$.

Pour tout $x, y \in \Lambda$ dans la même variété stable, on note $h^{s}(x, y): \mathcal{E}_{x} \rightarrow \mathcal{E}_{y}$ l'application d'holonomie du feuilletage stable-fort $\mathcal{F}^{s s}$. On note de même $h^{u}(x, z): \mathcal{E}_{x} \rightarrow \mathcal{E}_{z}$ l'application d'holonomie du feuilletage instable-fort, pour tout $z \in W^{u}(x)$.

Rappelons que pour un cocycle continu $F$ au-dessus d'une transformation $f:(X, \mu) \rightarrow$ $(X, \mu)$ on peut toujours trouver des mesures $F$-invariantes se projettant sur $\mu$ (voir la Remarque 1.4).

Notre premier but ici est de démontrer le Théorème 1 qui, avec les notations ci-dessus, s'exprime de la façon suivante :

ThÉORÈme 9. - Soit $(M, f, \mu, \mathcal{E}, F, m)$ un cocycle projectif mesuré tel que le cocycle $F$ est dominé et tous ses exposants de Lyapunov pour $\mu$ sont nuls.

Alors $m$ admet un désintegration $\left(\bar{m}_{x}\right)_{x}$ telle que

- $\bar{m}_{x}$ dépend continûment du point $x \in \Lambda$;

- pour tous points $x, y, z \in \Lambda$ tels que $y \in W^{s}(x)$ et $z \in W^{u}(x)$ on a $h^{s}(x, y)_{*} \bar{m}_{x}=$ $\bar{m}_{y}$ et $h^{u}(x, z)_{*} \bar{m}_{x}=\bar{m}_{z}$

- $\left(F_{x}\right)_{*} \bar{m}_{x}=\bar{m}_{f(x)}$ pour tout $x \in \Lambda$.

Pour tout point $x$ dans un ensemble basique hyperbolique $\Lambda$ on peut trouver des voisinages $R^{u}$ et $R^{s}$ de $x$, respectivement dans la variété stable et dans la variété instable de $x$, et un voisinage $R$ de $x$ dans $\Lambda$ qui est homéomorphe au produit $R^{u} \times R^{s}$, les verticales correspondant aux variétés stables locales et les horizontales aux variétés instables locales. On peut alors définir, pour une mesure quelconque dans $R$, ses projections sur $R^{u}$ et sur $R^{s}$.

Dans la démonstration du théorème on utilisera le fait que tout état d'équilibre $\mu$ d'un potentiel Hölder continu a la propriété de produit local : pour tout $x \in \lambda$ on peut trouver $R$ tel que $\mu \gg \mu^{u} \times \mu^{s}$, où $\mu^{u}$ et $\mu^{s}$ sont les projections de $\mu \mid R$ sur $R^{u}$ et $R^{s}$, respectivement. C'est à dire, $\mu(A \times B)>0$ pour tout $A \subset R^{u}$ et $B \subset R^{s}$ tels que $\mu^{u}(A)>0$ et $\mu^{s}(B)>0$. Voir $[2,12,19]$.

Les mêmes arguments qu' on a utilisés pour le Lemme 1.3 nous donnent

LEMME 2.10. - Avec les notations ci-dessus, pour tout ensemble $E \subset R$ de $\mu$-mesure totale dans $R$ il existe $x \in E$ tel que $E_{x}=\bigcup\left\{W_{\text {loc }}^{u}(y): y \in W_{\mathrm{loc}}^{s}(x) \cap E\right\}$ a aussi $\mu$-mesure totale dans $R$.

À présent nous démontrons le Théorème 9 :

Démonstration. - D'après le Théorème 8 , apliqué à $f$ et à $f^{-1}$, il existe un ensemble $E \subset \Lambda$ de $\mu$-mesure totale tel que

1. $m_{y}=h^{s}(x, y)_{*} m_{x}$ et $m_{z}=h^{u}(x, z)_{*} m_{x}$ pour tout $x, y, z \in E$ tels que $y \in W^{s}(x)$ et $z \in W^{u}(x)$;

2. $\left(F_{x}\right)_{*} m_{x}=m_{f(x)}$ pour tout $x \in E$.

Pour un point $w$ quelconque dans $\Lambda$ considérons un voisinage $R$ homéomorphe à un produit $R^{u} \times R^{s}$ comme dans la définition de la propriété de produit local. D'après le Lemme 1.3, on peut trouver $x \in E \cap R$ tel que $E_{x}=\bigcup\left\{W_{\mathrm{loc}}^{u}(y): y \in W_{\mathrm{loc}}^{s}(x) \cap E\right\}$ a $\mu$-mesure totale dans $R$. Au-dessus de $E_{x}$ les mesures conditionelles sont préservées par 
$\psi(x, z)=h^{u}(y, z) \circ h^{s}(x, y)$. On peut alors prolonger $\left(m_{z}\right)_{z \in E_{x}}$ de façon continue à tout le voisinage $R$, par l'expression $\bar{m}_{z, R}=\psi(x, z)_{*} m_{x}$.

De cette façon, nous pouvons construire un recouvrement de $\Lambda$ par des ouverts $R$ et des applications continues $z \mapsto \bar{m}_{z, R}$ définies sur chacun des ouverts, qui coïncident avec la désintégration $m_{x}$ sur un sous-ensemble de $\mu$-mesure totale. Ceci implique que sur $R_{1} \cap R_{2}$ ces applications continues coïncident sur un ensemble dense, et donc partout. On notera $\bar{m}_{z}=\bar{m}_{z, R}$ pour tout $z \in \Lambda$ et n'importe quel $R$ contenant $z$. La famille $\left(\bar{m}_{z}\right)_{z}$ est donc une désintégration continue pour la mesure $m$.

Comme $h^{s}(x, y), h^{u}(x, z)$ et $F_{x}$ dépendent continûment des points, les propriétés 1 et 2 ci-dessus se prolongent à tous les points de $\Lambda$, ce qui démontre l'invariance par holonomie et par itération.

On munit l'ensemble des cocycles projectifs de classe $C^{1}$ au-dessus de $f: M \rightarrow M$ de la topologie $C^{0}$. Fixons un atlas de $M$ par des cartes locales avec domaines compactes et au-dessus desquelles le fibré $\mathcal{E}$ est trivial. Pour toute constante $C>0$, on dira que la norme $C^{1}$ de $F$ est bornée par $C$ si dans chaque carte la dérivée de l'application $x \mapsto F_{x}$ est bornée par $C$ en norme.

Dans ce qui reste de cette section nous démontrons le résultat suivant, qui contient le Théorème 2 :

ThÉORÈme 10. - Pour chaque $C>0$ fixé, il existe un sous-ensemble ouvert dense par rapport à la topologie $C^{0}$, de l'espace des cocycles de classe $C^{1}$ dominés au-dessus de $f: \Lambda \rightarrow \Lambda$ dont la norme $C^{1}$ est bornée par $C$, tel que tout cocycle dans ce sousensemble a des exposants de Lyapunov non-nuls pour tout état d'équilibre $\mu$ de $f$ d'un potentiel hölderien.

Démonstration. - Commençons par remarquer que la conclusion est vraie s'il existe un point fixe (ou périodique) $p \in \Lambda$ et un point homocline $q \in \Lambda$ de $p$ tels que

1. toutes les valeurs propres de $F_{p}$ est hyperbolique sont de multiplicité 1 et avec modules différents ;

2. l'ensemble des directions propres $\left\{\xi_{1}, \ldots, \xi_{k}\right\}$ de $F_{p}$ est disjoint de l'ensemble $\left\{h^{u}(p, q) h^{s}(p, q) \xi_{i}: 1 \leqslant i \leqslant k\right\}$ de leurs images.

Ceci est un conséquence directe du Théorème 9 , en utilisant exactement les mêmes arguments que pour la démonstration du Théorème 7 . Ils nous suffit donc de démontrer que la condition ci-dessus est satisfaite par un sous-ensemble ouvert dense de cocycles.

Pour l'ouverture il suffit de remarquer que les holonomies stable et instable varient continûment avec le cocycle dans la topologie considèrée. En effet, d'autant que le cocycle reste Lipschitz avec constante de Lipschitz bornée, le cocycle relevé $\tilde{F}$ au-dessus du sous-shift est aussi uniformément Lipschitz. On conclut par les Lemmes 1.14 et 2.7.

Finalement, pour avoir la densité il suffit de perturber $F$, d'abord dans un voisinage du point $p$, pour avoir la condition 1 sur $F_{p}$, puis dans un voisinage du point $q$ qui évite tous les autres itérés de $q$, pour avoir la condition sur les holonomies.

Remarque 2.11. -

(a) La densité dans le Théorème 10 est valable dans toute topologie $C^{r}, 1 \leqslant r \leqslant \infty$.

(b) Ces arguments se généralisent de façon immédiate au cas des cocycles $(C, v)$ Hölder, avec constantes de Hölder fixés. Dans ce contexte la théorie de 
l'hyperbolicité normale ne s'applique plus, mais le Lemme 2.7 nous permet quand même de construire les holonomies stable et instable-forte.

(c) Théorème 10 reste valable si on permet de plus que la dynamique $f$ de la base varie dans la topologie $C^{1}$.

Remarque 2.12. - Théorème 10 reste valable aussi dans le cadre des cocycles dominées réels, c'est à dire que l'action dans les fibres vit dans $\operatorname{PSL}(k, \mathbb{R})$. La démonstration est analogue a celle du Corollaire 1.19.

Les mêmes arguments que dans la Section 1.6 montrent que, comme l'affirme la Proposition 0.1, les cocycles dont les exposants de Lyapunov sont nuls correspondent à un sous-ensemble de mesure de Lebesgue zéro dans l'espace des paramètres, pour un ouvert dense de familles paramétrées dans l'espace des cocycles dominés :

Proposition 2.13. - Il existe un ouvert dense de familles à $l \in \mathbb{N}$ paramètres $t \mapsto F_{t}$ de cocycles projectifs de classe $C^{r}, r \geqslant 1$ dominés au dessus de $f: \Lambda \rightarrow \Lambda$, pour lesquelles l'ensemble des $t$ tels que les exposants de Lyapunov de $F_{t}$ sont nuls pour un état d'équilibre d'un potentiel hölderien est discret, donc de mesure de Lebesgue nulle.

Démonstration. - La démonstration est analogue à celle du Corollaire 1.22. Il suffit de remarquer que, comme les normes $C^{r}$ sont plus fines que les normes $v$-Hölder (voir la Remarque 1.24) l'énoncé de différentiabilité donné par le Lemme 1.21 reste valable dans le cadre présent. En conséquence, les conditions 1 et 2 dans la démonstration du Théorème 10 correspondent à un sous-ensemble de codimension positive. De plus, comme dans la Remarque 1.23, si l'on fait varier le point périodique $p$ on obtient un sous-ensemble de codimension arbitrairement grande.

\section{Cocycles projectifs tangents à un feuilletage, au dessus d'un flot d'Anosov}

Dans cette section nous allons donner un cadre géométrique naturel où s'appliqueront les résultats sur les cocycles localement constants au-dessus d'un sous-shift de type fini : il s'agit des cocycles obtenus en relevant un flot d'Anosov transitif d'une variété compacte $M$ sur les feuilles d'un feuilletage transversalement projectif, transverse à une fibration $\pi: \mathcal{E} \rightarrow M$ de fibre $\mathbb{C P}^{k-1}$.

\subsection{Cocycles projectifs au dessus d'un flot}

Soit $V$ une variété et soit $\pi: \mathcal{E} \rightarrow V$ un fibré projectif admettant un feuilletage $\mathcal{F}$ sur $\mathcal{E}$ dont la dimension est égale à celle de $V$, et dont les feuilles sont transverses aux fibres. On suppose de plus que $\mathcal{F}$ est transversalement projectif c'est à dire que toutes les holonomies d'une fibre sur une autre sont des tranformations projectives. Un tel fibré s'appelle un fibré projectif plat.

Rappelons que la donnée d'un fibré plat et d'un tel feuilletage est équivalente à la donnée d'un homomorphisme contravariant $\rho: \pi_{1}(V) \rightarrow \operatorname{PSL}(k, \mathbb{C})$ (c'est à dire que $\left.\rho\left(\gamma_{1} \gamma_{2}\right)=\rho\left(\gamma_{2}\right) \cdot \rho\left(\gamma_{1}\right)\right)$ : en effet, $\rho$ est la représentation d'holonomie de $\mathcal{F}$; réciproquement étant donnée $\rho$ on construit $(\mathcal{E}, \mathcal{F})$ comme la suspension de $\rho$ (voir [16]); le fibré et le feuilletage sont alors $C^{\infty}$. 
Maintenant, à tout flot $X$ sur $V$ on peut associer son relevé $Y$ sur le feuilletage $\mathcal{F}$ : c'est l'unique flot dans les feuilles de $\mathcal{F}$, dont les trajectoires se projettent sur celles de $X$, en préservant le temps.

En résumé, à tout flot $X$ de $V$ et à toute représentation $\rho: \pi_{1}(V) \rightarrow P S L(k, \mathbb{C})$ nous avons associé un cocycle projectif $Y$ au dessus de $X$. Nous rappelons à présent l'énoncé du Théorème 3, dont la démonstration est l'objet de cette section :

THÉORÈME 11. - Soit $V$ une variété compacte, munie d'un flot d'Anosov transitif $X$ et soit $\pi: \mathcal{E} \rightarrow V$ un fibré projectif plat de fibre $\mathbb{C P}^{k-1}$. Soit $\mathcal{F}$ un feuilletage transverse aux fibres et transversalement projectif. Notons $Y$ le relevé de $X$ sur les feuilles de $\mathcal{F}$. Alors l'une des deux propriétés suivantes est vérifiée :

- Le feuilletage $\mathcal{F}$ admet une mesure transverse invariante par holonomie.

- Pour tout état d'équilibre $\mu$ de $X$ associé à un potentiel höldérien, le cocycle projectif induit par $Y$ au dessus de $X$ possède un exposant de Lyapunov non-nul.

\subsection{Dynamique symbolique et états d'équilibre d'un flot d'Anosov}

Cette section rappelle le formalisme symbolique introduit dans $[6,8]$ pour les flots vérifiant l'Axiome A.

Soit $\sigma: \Sigma_{T} \rightarrow \Sigma_{T}$ un sous-shift de type fini associé à une matrice $T$ à coefficients dans $\{0,1\}$. Soit $\left.\psi: \Sigma_{T} \rightarrow\right] 0,+\infty\left[\right.$ une fonction continue. On note $G=\left\{G_{t}: \Lambda(T, \psi) \rightarrow\right.$ $\Lambda(T, \psi)\}_{t \in \mathbb{R}}$, le flot obtenu comme suspension de $\sigma$ avec temps de retour égal à $\psi$.

Soit $X$ un flot d'Anosov transitif d'une variété compacte $V$. Il existe une matrice $T$ et une fonction Hölder continue $\left.\psi: \Sigma_{T} \rightarrow\right] 0,+\infty[$ et une surjection Hölder continue $P: \Lambda(T, \psi) \rightarrow V$ telle que :

- $P$ réalise une semi-conjugaison entre le flot $G$ et le flot $X$.

- Chaque cube $C_{i}=P(\{(x, t), x \in[0 ; i], t \in[0, \psi(x)]\})$ est l'adhérence de son intérieur.

- P est injective au dessus d'un sous-ensemble résiduel invariant de V qui, de plus, a mesure totale pour toute probabilité invariante ergodique qui charge les ouverts. On appelle partition de Markov une telle application, et rectangle toute image $R_{i}=$ $P([0 ; i] \times\{0\})$.

Étant donnée une partition de Markov $P: \Lambda(T, \psi) \rightarrow V$, on peut en construire d'autres par les procédures de raffinement suivantes : soit $s \in \mathbb{N}$

- raffinement de l'alphabet : on prend comme nouveaux symboles les $\xi=\left(i_{1}, \ldots, i_{s}\right)$ tels que $T_{i_{j}, i_{j+1}}=1$. La nouvelle matrice d'incidence est définie par $\tilde{T}_{\xi, \eta}=1$ si et seulement si $\xi$ et $\eta$ s'écrivent $\xi=\left(i_{1}, \ldots, i_{s}\right)$ et $\eta=\left(i_{2}, \ldots, i_{s+1}\right)$. L'application $\xi=\left(i_{1}, \ldots, i_{s}\right) \rightarrow i_{1}$ induit une conjugaison entre les sous-shifts associés à $T$ et $\tilde{T}$, et l'on définit $\tilde{\psi}$ comme la composée de $\psi$ par l'homéomorphisme de conjugaison.

- dédoublement de rectangles : les nouveaux symboles sont les $\xi=(i, t)$ avec $t \in\{0, \ldots, s-1\}$ et la matrice $\tilde{T}$ est définie par $\tilde{T}_{\xi, \eta}=1$ si et seulement si $\xi$ et $\eta$ s'écrivent $\xi=(i, t)$ et $\eta=(j, r)$ avec

$$
(i=j \text { et } r=t+1) \quad \text { ou } \quad\left(t=s-1, r=0 \text {, et } T_{i, j}=1\right) .
$$


LEMME 3.1. - Pour tout recouvrement fini $U_{l}$ de $V$ par des ouverts, il existe un sous-shift de type fini $\sigma: \Sigma_{T} \rightarrow \Sigma_{T}$, une fonction $\psi$ et une partition de Markov $P: \Lambda(T, \psi) \rightarrow V$ de $X$ tels que :

1. Pour tout cube $C_{j}$ il existe $I(j)$ tel que $C_{j} \subset U_{I(j)}$.

2. Pour tout $j$, il existe $L(j)$ tel que, pour tout $k$ vérifiant $T_{j, k}=1$, on a $I(k)=$ $L(j)$.

Démonstration. - Pour construire une partition de Markov vérifiant la condition 1, on choisit une partition de Markov quelconque $P_{0}$ et on choisit un raffinement $P_{1}: \Lambda\left(T_{1}, \psi_{1}\right) \rightarrow V$ de cette partition de façon que le diamètre de chaque cube soit plus petit que le nombre de Lebesgue du recouvrement $U_{l}$. Chaque cube $C_{j}$ est donc inclus dans un ouvert $U_{I_{1}(j)}$.

Soient $[0 ; i]$ les cylindres élémentaires de $P_{1}$. Notons $P_{2}: \Lambda\left(T_{2}, \psi_{2}\right) \rightarrow V$ le raffinement de $P_{1}$ consistant à prendre comme cylindres élémentaires les cylindres $[0 ; i, j]$ de $P_{1}$. Pour chaque nouveau symbole $\xi=(i, j)$ on note $I(\xi)=I_{1}(i)$ et $L(\xi)=I_{1}(j)$. Par définition $T_{\xi, \eta}=1$ si et seulement si $\xi$ et $\eta$ s'écrivent de la forme $\xi=(i, j)$ et $\eta=(j, k)$ ce qui implique $I(\eta)=L(\xi)$.

Soit $\phi: V \rightarrow \mathbb{R}$ une fonction Hölder continue. Rappelons que la construction de l'état d'équilibre du potentiel $\phi$ pour le flot $X$ est faite dans [8] de la façon suivante :

Soit $P: \Lambda(T, \psi) \rightarrow V$ une partition de Markov. La fonction $\tilde{\phi}: \Sigma_{T} \rightarrow \mathbb{R}$ définie par

$$
\tilde{\phi}(x)=\int_{0}^{\psi(x)} \phi \circ P(x, t) d t
$$

est encore Hölder continue. Il existe donc un unique état d'équilibre $\mu_{\sigma}$ du potentiel $\tilde{\phi}$ pour le sous-shift $\sigma: \Sigma_{T} \rightarrow \Sigma_{T}$. Alors $\mu_{G}=\left(1 / \int \psi d \mu_{\sigma}\right)\left(\mu_{\sigma} \times d t\right)$ est une mesure de probabilité invariante pour le flot $G$ sur $\Lambda(T, \psi)$. L'état d'équilibre sur $V$ est défini comme $\mu_{X}=P_{*}\left(\mu_{G}\right)$.

On conclut ce paragraphe en remarquant que tout cocycle projectif $Y$ au dessus du flot d'Anosov $X$ induit un cocycle projectif $\tilde{Y}$ au dessus du flot $G$ : le fibré $\tilde{\mathcal{E}}$ est le pullback par $P$ du fibré projectif $\mathcal{E}$ et $\tilde{Y}_{s}: \tilde{\mathcal{E}}_{(x, t)} \rightarrow \tilde{\mathcal{E}}_{G_{s}(x, t)}$ est (via l'identification canonique) l'application $Y_{s}: \mathcal{E}_{P(x, t)} \rightarrow \mathcal{E}_{X_{s}(P(x, t))}$, pour tout $s \in \mathbb{R}$.

On note $\mathcal{E}^{\sigma}$ la restriction de $\tilde{\mathcal{E}}$ au dessus de $\Sigma_{T} \simeq \Sigma_{T} \times\{0\}$, et $Y^{\sigma}$ le cocycle obtenu comme application de premier retour de $\tilde{Y}$ sur $\mathcal{E}^{\sigma}$. En d'autres termes $Y_{x}^{\sigma}: \mathcal{E}_{x}^{\sigma} \rightarrow \hat{\mathcal{E}}_{\sigma(x)}^{\sigma}$ coïncide avec la restriction de $\tilde{Y}_{\psi(x)}$ à $\tilde{\mathcal{E}}_{(x, 0)}$.

Remarque 3.2. - Pour tout cocycle projectif $(\mathcal{E}, Y)$ sur $V$ à valeurs dans $S L(k, \mathbb{C})$, et tout état d'équilibre $\mu_{X}$, l'existence d'exposants non-nuls est équivalente pour $\left(Y, \mu_{X}\right)$, pour $\left(\tilde{Y}, \mu_{G}\right)$ et pour $\left(Y^{\sigma}, \mu_{\sigma}\right)$; de plus les multiplicités des exposants sont les mêmes.

\subsection{Preuve du Théorème 11}

Soit $V$ une variété différentiable compacte munie d'un flot d'Anosov transitif $X$. Soit $\pi: \mathcal{E} \rightarrow V$ un fibré projectif muni d'un feuilletage $\mathcal{F}$ transverse aux fibres et transversalement projectif. Soit $Y$ le cocycle projectif au dessus de $X$ obtenu en relevant 
$X$ sur les feuilles de $\mathcal{F}$. Soit $\mu_{X}$ un état d'équilibre d'un potentiel hölderien pour le flot $X$. On suppose que les exposants de Lyapunov du cocycle $Y$ pour $\mu_{X}$ sont nuls et on montrera que les holonomies du feuilletage $\mathcal{F}$ laissent invariante une famille continue de probabilités dans les fibres.

On choisit un système de cartes $U_{l} \subset V$ couvrant $V$ de façon que les $\pi^{-1}\left(U_{l}\right)$ soient des cartes trivialisant le feuilletage $\mathcal{F}$ : il existe un difféomorphisme $\psi_{l}: \pi^{-1}\left(U_{l}\right) \rightarrow$ $U_{l} \times \mathbb{C P}^{k-1}$ se projetant par $\pi$ sur l'identité de $U_{l}$ et qui est projectif dans les fibres, tel que $\psi_{l}(\mathcal{F})$ est le feuilletage horizontal. Quitte à raffiner ce recouvrement, on peut supposer que pour tout couple $l_{1}, l_{2}$ l'intersection $U_{l_{1}} \cap U_{l_{2}}$ soit connexe. Alors, pour tout $l_{1}, l_{2}$ tels que $U_{l_{1}} \cap U_{l_{2}}$ soit non-vide, il existe une matrice $A_{l_{1}, l_{2}} \in \operatorname{PSL}(k, \mathbb{C})$ telle que le changement de carte $\psi_{l_{2}} \circ \psi_{l_{1}}^{-1}: U_{l_{1}} \cap U_{l_{2}} \times \mathbb{C P}^{k-1} \rightarrow U_{l_{1}} \cap U_{l_{2}} \times \mathbb{C P}^{k-1}$ soit défini $\operatorname{par}(x, z) \mapsto\left(x, A_{l_{1}, l_{2}}(z)\right)$.

Notons $P: \Lambda(T, \psi) \rightarrow V$ une partition de Markov associée par le Lemme 3.1 à ce recouvrement. Remarquons que pour tout cylindre [0;i], l'intersection $U_{I(i)} \cap U_{L(i)}$ est non-vide, car $U_{L(i)}$ contient l'extrémité d'un segment d'orbite inclus dans $U_{I(i)}$.

Lemme 3.3. - Avec les notations ci-dessus, le cocycle $Y^{\sigma}$, exprimé dans les cartes trivialisantes, est constant sur chaque cylindre $[0 ; i]$ de $\Sigma_{T}$.

Démonstration. - Voyons que $A_{I(i), L(i)}$ est la valeur du cocycle au-dessus de $[0 ; i]$. En effet, soit $x \in[0 ; i]$. Le point $P(x)$ appartient donc à $U_{I(i)}$ et cet ouvert contient tout le segment d'orbite de $P(x)$ pour les temps $t \in[0, \psi(x)]$. Dans la carte $\psi_{I(i)}$ le feuilletage est horizontal et l'expression du cocycle est donnée par l'identité sur les fibres. Audessus du point $Y_{\psi(x)}(P(x))$, la carte trivialisante de référence est $\psi_{L(i)}$. L'expression du cocycle dans les deux cartes de référence est donc $A_{I(i), L(i)}$.

Notons $\mu_{\sigma}$ l'état d'équilibre de $\sigma$ correspondant à $\mu_{X}$. Comme, par hypothèse, tout exposant de Lyapunov du cocycle $Y$ par rapport à $\mu_{X}$ est nul, il en est de même pour le cocycle $Y^{\sigma}$ par rapport à $\mu_{\sigma}$, d'après la Remarque 3.2. Comme on vient de le montrer, $Y^{\sigma}$, exprimé dans les cartes trivialisantes $\left(U_{l}, \psi_{l}\right)$, est localement constant, engendré par les $A_{i}=A_{I(i), L(i)}$. D'après les Corollaires 1.7 et 1.8 , il existe alors des probabilités $\left\{\theta_{1}, \ldots, \theta_{n}\right\}$ sur $\mathbb{C} \mathbb{P}^{k-1}$ telles que $\left(A_{i}\right)_{*}\left(\theta_{i}\right)=\theta_{j}$ pour tout $i, j$ tels que $T_{i, j}=1$. On en déduit qu'il existe une famille $\left\{\theta_{\xi}\right\}_{\xi \in \Lambda(T, \psi)}$ de probabilités sur les fibres de $\tilde{\mathcal{E}}$, invariante par l'action de $\tilde{Y}$ et cö̈ncidant avec $\theta_{i}$ sur $[0 ; i] \times\{0\}$ (dans les cartes $\left(U_{I(i)}, \psi_{I(i)}\right)$ ).

Dans le prochain énoncé on note $W^{c s}(x)$ la variété centre-stable de $x$, c'est à dire, l'ensemble des points dont les orbites positives sont asymptotiques à l'orbite positive de $x$. D'autre part, on note $W^{s}(x)$ la variété stable de $x$, définie comme l'ensemble des points le $t$-ème iteré est asymptotique au $t$-ème iteré de $x$ quand $t \rightarrow+\infty$. Rappelons que ces ensembles sont des sous-variétés immergées, aussi differentiables que le flot $X$, et que $W^{c s}(x)$ est l'union des variétés stables des points de l'orbite de $x$. De plus, tout chemin dans $W^{c s}(x)$ joignant $x$ à un point $y$ est homotope, dans $W^{c s}(x)$ et à extrémités fixées, au produit d'un chemin sur l'orbite de $x$ et un chemin sur la variété stable de $y$.

La famille des variétés centre-stables, respectivement stables, forme le feuilletage centre-stable $\mathcal{F}^{c s}$, respectivement, stable $\mathcal{F}^{s}$, du flot $X$. On définit de même les variétés et les feuilletages centre-instables ou instables. 
LEMME 3.4. - Il existe un ensemble $E \subset V$ invariant par le flot $X$ et de $\mu_{X}$-mesure totale, et il existe une famille $\left(\theta_{x}\right)_{x \in E}$ de probabilités dans les fibres $\mathcal{E}_{x}$, telle que

- la famille est invariante par l'action du flot $Y$ dans les fibres ;

- pour tout $x, y \in E$ tels que $y \in W^{c s}(x)$ (respectivement $y \in W^{c u}(x)$ ), et pour tout chemin $\gamma$ joignant $x$ et $y$ dans $W^{c s}(x)$ (respectivement dans $W^{c u}(x)$ ) on a $\left(H_{\gamma}\right)_{*}\left(\theta_{x}\right)=\theta_{y}$, où $H_{\gamma}$ est l'holonomie du feuilletage $\mathcal{F}$ au-dessus de $\gamma$.

Démonstration. - Notons $W \subset V$ le lieu d'injectivité de la partition de Markov $P$. Rappelons qu'il est invariant par $X$ et de $\mu_{X}$-mesure totale. On peut alors définir une famille de probabilités $\left\{\theta_{x}\right\}_{x \in W}$ sur les fibres $\mathcal{E}_{x}$ invariante par l'action du flot de $Y$ : il suffit de prendre $\theta_{x}=\theta_{\xi}$ où $\xi=P^{-1}(x)$ (modulo l'identification canonique des fibres $\mathcal{E}_{x}$ et $\left.\tilde{\mathcal{E}}_{\xi}\right)$.

De plus par construction, si $x, y \in W$ sont dans un même cube $C_{i}$, pour tout chemin $\gamma$ joignant $x$ à $y$ dans l'ouvert $U_{I(i)}$ l'image de $\theta_{x}$ par l'holonomie $H_{\gamma}$ du feuilletage $\mathcal{F}$ au dessus de $\gamma$ est $\theta_{y}$. En effet, ceci revient à dire que la famille $\left(\theta_{z}\right)_{z}$ est constante sur $C_{i}$ dans la carte $\left(U_{I(i)}, \psi_{I(i)}\right)$. Sur le rectangle $R_{i}$ c'est une conséquence de la construction dans le paragraphe précédent : dans la carte trivialisanet la probabilité est donnée par $\theta_{i}$. Cela se prolonge à $C_{i}$ par invariance par le flot $Y$.

Nous définissons $E$ comme l'ensemble des points de $W$ dont les orbites positives et négatives sont denses dans $\Lambda$. Clairement, il s'agit d'un ensemble invariant par $X$ et, comme $\mu_{X}$ est ergodique et charge les ouverts, $E$ a $\mu$-mesure totale. Il nous reste à montrer que la famille $\left(\theta_{x}\right)_{x \in E}$ est invariante par holonomie au-dessus des chemins inclus dans des variétés centre-stables (ou centre-instables). La preuve est analogue à celle du Théorème 8 .

Soit $x, y \in E$ tels que $y \in W^{c s}(x)$, et soit $\gamma \subset W^{c s}(x)$. Si $\gamma$ est inclus dans l'orbite de $x$, l'invariance par holonomie le long de $\gamma$ découle de l'invariance par le flot $Y$. L'holonomie ne dépendant que de la classe d'homotopie du chemin, d'après la remarque précédant le lemme on peut maintenant supposer que $\gamma$ est inclus dans la variété stable de $x$. Comme la famile $\left(\theta_{z}\right)_{z}$ est invariante par le flot, on a le droit de remplacer $\gamma, x$, $y$ par des itérés quelconques. Comme l'orbite positive de $x$ est dense dans $\Lambda$, et que la longueur des itérés de $\gamma$ tend vers zéro, on peut trouver des itérés inclus dans l'intérieur d'un même cube $C_{i}$. On conclut alors par l'invariance par holonomie au dessus de chaque cube $C_{i}$ : voir le deuxième paragraphe de cette preuve.

Dans la suite on utilisera le fait que tout état d'équilibre $\mu_{X}$ d'un flot d'Anosov a la propriété de produit local : pour toute section $S$ transverse au flot, la mesure $\mu_{S}$ induite par $\mu_{X}$ dans $S$ a la propriété de produit local pour les feuilletages $\mathcal{F}_{S}^{S}$ et $\mathcal{F}_{S}^{u}$ induits sur $S$ par intersection des feuilletages centre-stable et centre-instable du flot. Voir $[2,12,19]$.

Le prochain corollaire conclut la démonstration du Théorème 11 :

Corollaire 3.5. - Il existe une famille continue de probabilités $\left(\bar{\theta}_{x}\right)_{x \in V}$ sur les fibres $\mathcal{E}_{x}$, définie sur toute la variété $V$, et cö̈ncidant avec la famille $\left(\theta_{x}\right)_{x \in E}$ sur un sousensemble de $\mu_{X}$-mesure totale. De plus, la famille $\left(\bar{\theta}_{x}\right)_{x \in V}$ est invariante par l'action du flot $Y$ et de l'holonomie du feuilletage $\mathcal{F}$.

Démonstration. - Pour tout point $z \in V$ on fixe une petite section transverse $S$ du flot $X$ au point $z$. Comme $E$ est un ensemble $X$-invariant de $\mu_{x}$-mesure totale, il intersecte 
$S$ dans un ensemble de $\mu_{S}$-mesure totale. Comme dans la preuve du Théorème 9 , on peut alors trouver une famille continue $\left(\bar{\theta}_{x}\right)_{x \in S}$ définie sur toute la section transverse $S$ et coïncidant avec la famille $\left(\theta_{x}\right)_{x \in E \cap S}$ sur un sous-ensemble de $\mu_{S}$-mesure totale. Ce prolongement est unique ce qui permet de montrer qu'il est invariant pour toute holonomie des feuilletages $\mathcal{F}_{S}^{s}$ et $\mathcal{F}_{S}^{u}$. On prolonge alors $\left(\bar{\theta}_{x}\right)_{x \in S}$ à un voisinage du point $z$, par invariance sous l'action du flot $X$. Ceci définit un prolongement continu qui coïncide avec la famille $\left(\theta_{x}\right)_{x \in E}$ sur un sous-ensemble de $\mu_{X}$-mesure totale de ce voisinage. Ce prolongement est donc unique. On en déduit l'existence d'une famille continue $\left(\bar{\theta}_{x}\right)_{x \in V}$ définie sur toute la variété $V$, et coïncidant avec $\left(\theta_{x}\right)_{x \in E}$ sur un sousensemble de $\mu_{X}$-mesure totale.

D'après le Lemme 3.4, la famille $\left(\theta_{x}\right)_{x \in E}$ est invariante par l'action du flot et par les holonomies de $\mathcal{F}$ au-dessus des feuilles centre-stables et centre-instables. On en déduit, par continuité, que ces propriétés restent vraies pour $\left(\bar{\theta}_{x}\right)_{x \in V}$ : elle est invariante par toutes les holonomies du feuilletage $\mathcal{F}$ au-dessus des chemins inclus dans des variétés centre-stables ou centre-instables. Tout chemin étant homotope au produit de chemins inclus, respectivement, dans des feuilles des feuilletages $\mathcal{F}^{c s}$ et $\mathcal{F}^{c u}$, ceci montre que $\left(\bar{\theta}_{x}\right)_{x \in V}$ est invariante par toute holonomie de $\mathcal{F}$.

\subsection{Mesures SRB pour le flot relevé}

Le but de ce paragraphe est de montrer la Proposition 0.2.

Notons $\mu$ la mesure SRB du flot d'Anosov $X$. On suppose que le plus grand exposant de Lyapunov pour la mesure $\mu$ du cocycle défini par le flot $Y$ est de multiplicité 1 . Soit $x$ un point générique pour $\mu$. On note $\sigma(x)$ le point de l'espace projectif $\mathcal{E}_{x}$ qui représente la droite qui est l'espace de Lyapunov correspondant au plus grand exposant de $\mu$, et $\Sigma_{x} \subset \mathcal{E}_{x}$ l'hyperplan projectif correspondant aux autres exposants de Lyapunov. Remarquons que $\sigma: M \rightarrow \mathcal{E}$ est une section mesurable invariante par l'action des flots $X$ et $Y$. De plus, on vérifie facilement que, pour tout point $\xi \in \mathcal{E}_{x} \backslash \Sigma_{x}$ la distance

$$
d\left(Y_{t}(\xi), \sigma\left(X_{t}(x)\right)\right) \rightarrow 0
$$

quand $t$ tend vers $+\infty$.

Notons $v=\sigma_{*}(\mu)$; c'est une mesure de probabilité invariante ergodique pour $Y$. La Proposition 0.2 est une conséquence immédiate de

Proposition 3.6. - Avec les notations ci-dessus, v est une mesure SRB pour le flot $Y$ et son bassin d'attraction est de mesure de Lebesgue totale dans $\mathcal{E}$.

Démonstration. - Il nous suffit de montrer qu'il existe un sous-ensemble $B \subset \mathcal{E}$, de mesure de Lebesgue totale, tel que

$$
\lim _{T \rightarrow \infty} \frac{1}{T} \int_{0}^{T} \varphi\left(Y_{t}(\xi)\right) d t=\int \varphi d v
$$

pour $\xi \in B$ et pour un ensemble dénombrable dense de fonctions continues $\varphi$. Nous fixons un tel ensemble $\left\{\varphi_{l}: \mathcal{E} \rightarrow \mathbb{R}\right\}$. 
Comme les fonctions $\varphi_{l} \circ \sigma$ sont bornées, donc intégrables, le théorème ergodique de Birkhoff assure que, pour tout $l \in \mathbb{N}$, il existe un sous-ensemble $D_{l} \subset M$ de $\mu$-mesure totale tel que

$$
\lim _{T \rightarrow+\infty} \frac{1}{T} \int_{0}^{T}\left(\varphi_{l} \circ \sigma\right)\left(X_{t}(x)\right) d t=\int\left(\varphi_{l} \circ \sigma\right) d \mu
$$

pour tout $x \in D_{l}$. Soit $D=\bigcap_{l} D_{l}$ et $A$ l'union des $\mathcal{E}_{x} \backslash \Sigma_{x}$ pour $x \in D$. Le choix de $D$ et la propriété (17) impliquent que $A$ est contenu dans le bassin d'attraction de $v$ : pour tout $l$ et tout $\xi \in A$ on a

$$
\lim _{T \rightarrow+\infty} \frac{1}{T} \int_{0}^{T} \varphi_{l}\left(Y_{t}(\xi)\right) d t=\lim _{T \rightarrow+\infty} \frac{1}{T} \int_{0}^{T} \varphi_{l}\left(\sigma\left(X_{t}(\pi(\xi))\right)\right) d t=\int\left(\varphi_{l} \circ \sigma\right) d \mu=\int \varphi_{l} d \nu .
$$

Maintenant, pour chaque point $\xi \in A$, considérons le relèvement $W(\xi)$ de la variété stable du point $x=\pi(\xi)$ à la feuille du feuilletage $\mathcal{F}$ qui passe par $\xi$. Notons $B$ l'union des $W(\xi)$ sur tous les points $\xi \in A$. Il est clair que $B$ est encore inclus dans le bassin d'attraction de $v$ : les orbites positives de tous les points dans un même $W(\xi)$ sont asymptotiques, elles ont donc les mêmes moyennes de Birkhoff pour toute fonction continue.

Remarquons que la projection de $B$ contient l'union $C$ des variétés stables des points de $D$. Nous utilisons la propriété suivante des mesures SRB des systèmes d'Anosov (voir [7]) : pour tout ensemble de $\mu$-mesure totale, l'union des variétés stables de ses points a mesure de Lebesgue totale dans $M$. Il s'en suit que $C$ a mesure de Lebesgue totale dans $M$. D'autre part, comme $\mathcal{E}_{x} \backslash \Sigma_{x}$ a mesure de Lebesgue totale dans la fibre $\mathcal{E}_{x}$ et que le feuilletage $\mathcal{F}$ est projectif, l'ensemble $B$ contient un sous-ensemble de mesure de Lebesgue totale dans la fibre au-dessus de tout point de $C$. On en déduit que $B$ a mesure de Lebesgue totale dans $\mathcal{E}$, ce qui conclut.

\section{Feuilletages définis comme suspension : généricité des exposants de Lyapunov non-nuls}

\subsection{Suspension d'une représentation}

Nous rappelons ici brièvement la contruction des feuilletages définis par une suspension (voir [16]) :

Soit $V$ une variété compacte et $P: \tilde{V} \rightarrow V$ son revêtement universel. Notons $\rho_{0}: \pi_{1}(V) \rightarrow \operatorname{Aut}(P)$ la représentation (covariante) canonique dans le groupe des automorphismes du revêtement. Soit $\rho: \pi_{1}(V) \rightarrow \operatorname{PSL}(k, \mathbb{C})$ une représentation contravariante. On considère le quotient de $\tilde{V} \times \mathbb{C P}^{k-1}$ sous l'action du groupe fondamental $\pi_{1}(S)$ définie par $(z, w) \mapsto\left(\rho_{0}(\gamma) \cdot z, \rho(\gamma)^{-1} \cdot w\right)$. Le quotient de $\tilde{V} \times \mathbb{C} \mathbb{P}^{k-1}$ par cette action est un fibré projectif $\pi_{\rho}: V_{\rho} \rightarrow V$ de fibre $\mathbb{C P}^{k-1}$, la projection étant induite par la projection naturelle de $\tilde{V} \times \mathbb{C P}^{k-1}$ sur le premier facteur. Enfin le feuilletage horizontal de $\tilde{V} \times \mathbb{C P}^{k-1}$ dont les feuilles sont les $\tilde{V} \times\{w\}$ passe au quotient sur $V_{\rho}$ en un feuilletage $\mathcal{F}_{\rho}$ transverse aux fibres et transversalement projectif, dont la représentation 
d'holonomie (sur la fibre au dessus du point base de $V$ ) est exactement $\rho$. Remarquons que chaque feuille de $\mathcal{F}_{\rho}$ est un revêtement de $V$ et, par construction, admet $\tilde{V}$ comme revêtement universel.

On suppose à présent $V$ muni d'une métrique riemannienne $\|\cdot\|$ et on munit les feuilles de $\mathcal{F}_{\rho}$ de la métrique qui fait de la projection $\pi_{\rho}$ une isométrie locale : en particulier toute géodésique d'une feuille de $\mathcal{F}_{\rho}$ se projette par $\pi_{\rho}$ sur une géodésique de $V$.

Notons $T_{1}(V)$ et $T_{1}\left(\mathcal{F}_{\rho}\right) \subset T_{1}\left(V_{\rho}\right)$ le fibré unitaire tangent à $V$ et le fibré unitaire tangent du feuilletage $\mathcal{F}_{\rho}$, respectivement. La différentielle de la projection $\pi_{\rho}$ induit une projection naturelle $\left(\pi_{\rho}\right)_{*}: T_{1}\left(\mathcal{F}_{\rho}\right) \rightarrow T_{1}(V)$ de fibre $\mathbb{C P}^{k-1}$. De plus les fibrés unitaires tangents des feuilles de $\mathcal{F}_{\rho}$ sont les feuilles d'un feuilletage $\tilde{\mathcal{F}}_{\rho}$ sur $T_{1}\left(\mathcal{F}_{\rho}\right)$ dont les feuilles sont transverses aux fibres de $\left(\pi_{\rho}\right)_{*}$. Pour chaque feuille de $\mathcal{F}_{\rho}$, son flot géodésique est un flot, sur la feuille correspondante de $\tilde{\mathcal{F}}_{\rho}$, qui se projette sur le flot géodésique de $V \operatorname{par}\left(\pi_{\rho}\right)_{*}$. On a donc montré :

Remarque 4.1. - Le flot géodésique tangent aux feuilles de $\mathcal{F}_{\rho}$ est le relevé $Y=X_{\rho}$ sur les feuilles de $\tilde{\mathcal{F}}_{\rho}$ du flot géodésique $X$ de $V$. En particulier c'est un cocycle projectif au dessus de $X$.

On suppose à présent que le flot géodésique $X$ de $V$ est un flot d'Anosov transitif sur $T_{1}(V)$ qui laisse alors invariante la mesure de Liouville $\mu$, celle-ci ergodique (cette mesure est de plus un état d'équilibre pour $X$ ). Pour cela il suffit que la métrique $\|\cdot\|$ soit à courbure sectionnelle strictement négative [14,1].

Nous pouvons à présent conclure la démonstration du Théorème 4 qui est un cas particulier du résultat suivant :

THÉORÈme 12. - Avec les hypothèses sur $V$ ci-dessus, soit $\rho: \pi_{1}(V) \rightarrow \operatorname{PSL}(k, \mathbb{C})$ un morphisme contravariant du groupe fondamental. Alors l'une des propriétés suivantes est vérifiée:

- ou bien il existe une probabilité $\theta$ sur $\mathbb{C P}^{k-1}$ invariante par les transformations $\rho(\gamma)$, pour tout $\gamma \in \pi_{1}(V)$,

- ou bien le cocycle projectif défini par $X_{\rho}$ au-dessus de X possède des exposants de Lyapunov non-nuls pour la mesure de Liouville.

Démonstration. - D'après le Théorème 11 , si tous les exposants de Lyapunov de la mesure de Liouville sont nuls pour le cocycle $Y$ alors il existe une mesure de probabilité invariante par le groupe d'holonomie du feuilletage $\tilde{\mathcal{F}}_{\rho}$. Ce groupe d'holonomie est le même que celui de $\mathcal{F}_{\rho}$, ce qui conclut.

\subsection{Généricité de l'absence de mesures transverses invariantes}

Le Théorème 11 dit que si $V$ est une variété compacte portant un flot d'Anosov $X$ et si $\rho: \pi_{1}(V) \rightarrow P S L(k, \mathbb{C})$ est une représentation alors :

1. ou bien l'action naturelle de $\rho\left(\pi_{1}(V)\right)$ sur $\mathbb{C P}^{k-1}$ laisse invariante une probabilité,

2. ou bien tout état d'équilibre de $X$ possède des exposants de Lyapunov non-nuls pour le cocycle projectif au dessus de $X$ obtenu en relevant $X$ sur les feuilles du feuilletage $\mathcal{F}_{\rho}$, suspension de $\rho$. 
Dans cette section nous cherchons à comprendre en quelle mesure la possibilité décrite dans l'item 1 est rare, est donc en quelle mesure l'existence d'exposants de Lyapunov non-nuls est générique. On est donc amené à perturber les représentations $\rho$ afin d'interdire l'existence de probabilité invariante. La principale difficulté du problème est contenue dans les propriétés algébriques du groupe $\pi_{1}(V)$.

Nous présentons ici un exemple simple, où nous savons perturber la représentation : il s'agit du cadre du Théorème 4, quand la base est une surface compacte $S$ de genre $g \geqslant 2$ et que la fibre est $\mathbb{C P}^{1}$. On veut montrer que l'on peut perturber toute représentation $\rho: \pi_{1}(S) \rightarrow \operatorname{PSL}(2, \mathbb{C})$ de façon que le feuilletage $\mathcal{F}_{\rho}$ suspension de $\rho$ n'admette pas de mesure transverse invariante par holonomie.

On rappelle que la topologie naturelle sur l'espace des représentations consiste à dire que deux représentations sont proches si les matrices associées aux éléments d'un système fini de générateurs sont proches. Plus précisément, fixons $\left(\alpha_{1}, \beta_{1}, \ldots, \alpha_{g}, \beta_{g}\right)$ une famille génératrice du groupe fondamental $\pi_{1}(S)$ de la surface $S$ de genre $g \geqslant 2$, telle que $\pi_{1}(S)$ soit le quotient du groupe libre engendré par les $\left(\alpha_{i}, \beta_{i}\right)$ par l'unique relation $\left[\alpha_{1}, \beta_{1}\right] \cdots\left[\alpha_{g}, \beta_{g}\right]=1$. Posons

$$
V_{g}:=\left\{\rho=\left(A_{1}, B_{1}, \ldots, A_{g}, B_{g}\right) \in S L(2, \mathbb{C})^{2 g} \mid\left[A_{1}, B_{1}\right] \ldots\left[A_{g}, B_{g}\right]= \pm \operatorname{Id}\right\} .
$$

C'est une sous-variété algébrique complexe de $S L(2, \mathbb{C})^{2 g}$, qui paramétrise toutes les représentations $\rho: \pi_{1}(S) \rightarrow P S L(k, \mathbb{C})$, et la topologie naturelle de cette variété coïncide avec la topologie naturelle des représentations $\rho$.

Le but de cette partie est de montrer le Théorème 5 qui, avec les notations ci-dessus, s'exprime de la façon suivante :

THÉORÈME 13. - L'ensemble des représentations $\rho \in V_{g}$ ne laissant invariante aucune probabilité de $\mathbb{C} \mathbb{P}^{k-1}$ forme un ouvert dense dans $V_{g}$, pour $g \geqslant 2$.

Démonstration. - L'ensemble $\mathcal{R}$ des représentations qui laissent invariante une probabilité de $\mathbb{C P}^{k-1}$ est fermé dans $V_{g}$ (car l'ensemble des probabilités de $\mathbb{C P}^{k-1}$ est compact pour la topologie faible); nous avons donc juste besoin de montrer la densité de l'ensemble des représentations ne laissant invariante aucune probabilité sur $\mathbb{C P}^{1}$.

Notons $\Delta \subset S L(2, \mathbb{C})^{2 g}$ l'ensemble des $2 g$-uples de matrices tels qu'il existe deux droites (éventuellement confondues) dont l'union est invariante par chacune des matrices. Cet ensemble est fermé dans $S L(2, \mathbb{C})^{2 g}$, car l'ensemble des couples de droites est compact.

Notons NonHyp la sous-variété analytique réelle de $S L(2, \mathbb{C})$ formée des matrices dont les valeurs propres sont de module 1 . On vérifie que $A \notin$ NonHyp est équivalent à ce que l'application induite $A: \mathbb{C P}^{1} \rightarrow \mathbb{C P}^{1}$ ait deux points fixes hyperboliques, l'un attracteur et l'autre répulseur.

LEMME 4.2. - $\mathcal{R}$ est inclus dans $\Delta \cup$ NonHyp $^{2 g}$.

Démonstration. $-\mathrm{Si} \rho \in \mathcal{R}$ et $\rho \notin$ NonHyp $^{2 g}$, alors l'une des composantes $A_{i}$ ou $B_{i}$ de $\rho$ est hyperbolique, et les seules probabilités de $\mathbb{C P}^{1}$ invariantes par $\rho$ sont des barycentres des mesures de Dirac correspondant aux directions propres de cette matrice. Alors, ou bien l'une des directions propres est laissée invariante par chacun des $A_{j}, B_{j}$, 
ou bien l'union des directions propres est laissé invariant par les $A_{j}, B_{j}$ : dans chacun de ces cas $\rho \in \Delta$.

Pour montrer que $\mathcal{R}$ est d'intérieur vide dans $V_{g}$, il suffit donc de montrer que $V_{g} \cap \Delta$ et $V_{g} \cap$ NonHyp $^{2 g}$ sont d'intérieur vide dans $V_{g}$ (l'union de deux fermés d'intérieur vide est d'intérieur vide).

Soit $W$ une composante irréductible de $V_{g}$. La variété $V_{g}$ est définie par 3 équations, et donc chacune des composantes irréductibles $W$ de $V_{g}$ est de codimension (complexe) au plus 3 dans $S L(2, \mathbb{C})^{2 g}$, donc de dimension supérieure à $6 g-3$.

LEMME 4.3. - L'ensemble $\Delta$ est un sous-ensemble algébrique de dimension inférieure à $4 g+1$, et $\Delta \cap V_{g}$ est de dimension inférieure à $4 g<6 g-3$. En conséquence, $\Delta \cap V_{g}$ est d'intérieur vide dans $V_{g}$.

Démonstration. - Remarquons d'abord que $\Delta$ est la projection sur $\operatorname{PSL}(2, \mathbb{C})^{2 g} \mathrm{du}$ sous-ensemble de $\mathbb{C P}^{1} \times \mathbb{C P}^{1} \times P S L(2, \mathbb{C})^{2 g}$ défini par le fait que les matrices doivent préserver l'union des deux droites. C'est donc une projection propre d'un ensemble algébrique, donc un ensemble algébrique.

On écrit $\Delta=\Delta_{1} \cap \Delta_{2}$ où $\Delta_{1}$ est l'ensemble des $2 g$-uples de matrices préservant une droite, et où $\Delta_{2}$ est l'ensemble des $2 g$-uples de matrices préservant l'union de deux droites distinctes.

Pour tout couple $D_{1}, D_{2}$ de droites de $\mathbb{C}^{2}$ distinctes, notons $\Delta\left(D_{1}, D_{2}\right)$ l'ensemble des matrices préservant l'union de ces droites : on vérifie simplement que c'est un sous-ensemble algébrique de $S L(2, \mathbb{C})$ de dimension 1 . Remarquons que $\Delta_{2}=$ $\bigcup_{\left(D_{1} \neq D_{2}\right)}\left(\Delta\left(D_{1}, D_{2}\right)^{2 g}\right)$. Son adhérence est donc un ensemble algébrique de $\operatorname{SL}(2, \mathbb{C})^{2 g}$ de dimension $2 g+2$ qui contient l'ensemble des représentations $\rho$ possédant une matrice hyperbolique et préservant une mesure non-réduite à une mesure de Dirac. Sa dimension est $2 g+2<6 g-3 \leqslant \operatorname{dim} W$.

Pour toute droite $D$, notons $\Delta_{D}$ l'ensemble des matrices de $S L(2, \mathbb{C})$ laissant $D$ invariante. C'est un espace de dimension 2. On en déduit que l'ensemble $\Delta_{1}$ des représentations $\rho$ qui préservent une même droite est inclus dans un sous-espace $\Delta_{g} \subset$ $S L(2, \mathbb{C})^{2 g}$ de dimension $4 g+1$ qui est l'union sur les droites $D$ de $\Delta_{D}^{2 g}$.

\section{LEMME 4.4. - Pour toute droite $D, V_{g} \cap \Delta_{D}^{2 g}$ est d'intérieur vide dans $\Delta_{D}^{2 g}$.}

Démonstration. - Pour cela il suffit de remarquer que, pour tout couple $A=\left(\begin{array}{cc}\alpha & \delta \\ 0 & \alpha^{-1}\end{array}\right)$ et $B=\left(\begin{array}{cc}\beta & \gamma \\ 0 & \beta^{-1}\end{array}\right)$, on peut trouver un couple $\tilde{A}=\left(\begin{array}{cc}\tilde{\alpha} & \tilde{\delta} \\ 0 & \tilde{\alpha}^{-1}\end{array}\right)$ et $\tilde{B}=\left(\begin{array}{cc}\tilde{\beta} & \tilde{\gamma} \\ 0 & \tilde{\beta}^{-1}\end{array}\right)$, aussi proches que l'on veut de $A$ et de $B$, tel que $[A B] \neq[\tilde{A} \tilde{B}]$. En effet ce commutateur est de la forme $C=\left(\begin{array}{ll}1 & \omega \\ 0 & 1\end{array}\right)$ où $\omega$ est une fonction rationnelle non constante de $\alpha, \beta, \delta, \gamma$.

Ceci montre que l'on peut changer le commutateur $\left[A_{1}, B_{1}\right]$ tout en gardant l'invariance de la droite $D$. Ceci permet de briser la relation $\left[A_{1}, B_{1}\right]^{-1}=\prod_{2}^{g}\left[A_{i}, B_{i}\right]$.

On en déduit que $V_{g} \cap \Delta_{1}$ est d'intérieur vide dans $\Delta_{1}$, donc est de dimension inférieure à $4 g<6 g-3$, ce qui conclut la preuve du Lemme 4.3.

Considérons les projections $\pi_{j}: S L(k, \mathbb{C})^{2 g} \rightarrow S L(2, \mathbb{C})$ qui associent à tout $2 g$-uples de matrices sa $j$ ème-composante. 
LEMme 4.5. - Pour toute composante irréductible $W$ de $V_{g}$, et tout ouvert $U$ de $W$ il existe une projection $\pi_{i}(U)$ qui n'est pas incluse dans NonHyp.

Démonstration. - Soit $p \in U$ un point générique. L'espace tangent $T_{p}(W)$ est de même dimension que $W$, donc de codimension au plus 3 . Comme $2 g$ est plus grand que 3 , il existe $i$ telle que $\pi_{i}$ soit une submersion au point $p$. On en déduit que $\pi_{i}(U)$ n'est pas inclus dans NonHyp car celui-ci est d'intérieur vide dans $\operatorname{SL}(2, \mathbb{C})$.

Nous avons montré que $V_{g} \cap \Delta$ et $V_{g} \cap \mathrm{NonHyp}^{2 g}$ sont d'intérieur vide, ce qui conclut la preuve du Théorème 13.

\section{Remerciements}

Nous remercions Véronique Maume qui nous a expliqué le Théorème de Furstenberg ainsi que ses généralisations par Guivarc'h et Royer, ainsi que François Ledrappier pour les discussions qui ont permis d'affermir certains points clef de ce travail.

\section{RÉFÉRENCES}

[1] D. Anosov, Geodesic flows on closed Riemannian manifolds with negative curvature, Proc. Steklov Inst. Math. 90 (1967).

[2] M. Babillot, F. Ledrappier, Geodesic paths and horocyclic flow on abelian covers, in : Lie Groups and Ergodic Theory (Mumbai 1996), in : Tata Inst. Fund. Res. Stud. Math., Vol. 14, 1998, pp. 1-32.

[3] J. Bochi, Genericity of zero Lyapunov exponents, Ergodic Theory Dynamical Systems 22 (2002).

[4] C. Bonatti, X. Gómez-Mont, R. Vila, The foliated geodesic flow of Ricatti equations, Prepublication Dijon.

[5] C. Bonatti, X. Gómez-Mont, Sur le comportement statistique des feuilles de certains feuilletages holomorphes, Enseignement Mathématique 38 (2001).

[6] R. Bowen, Symbolic dynamics for hyperbolic flows, Amer. J. Math. 95 (1973) 428-459.

[7] R. Bowen, Equilibrium States and the Ergodic Theory of Anosov Diffeomorphisms, in: Lect. Notes in Math., Vol. 470, Springer-Verlag, 1975.

[8] R. Bowen, D. Ruelle, The ergodic theory of Axiom A flows, Invent. Math. 29 (1975) 181202.

[9] Ya. Brin, Ya. Pesin, Partially hyperbolic systems, Akad. Nauk SSSR 1 (1974) 170-212.

[10] H. Furstenberg, Noncommuting random products, Trans. Amer. Math. Soc. 108 (1963) 377428.

[11] Y. Guivarc'h, A. Raugi, Products of random matrices : convergence theorems, Contemp. Math. 50 (1986) 31-54.

[12] N. Haydn, Canonical product structure of equlibrium states, Rand. Comput. Dynam. 2 (1994) 79-96.

[13] M. Hirsch, C. Pugh, M. Shub, Invariant Manifolds, in: Lect. Notes in Math., Vol. 583, Springer-Verlag, 1977.

[14] E. Hopf, Ergodic theory and the geodesic flow on surfaces on constante negative curvature, Bull. Amer. Math. Soc. 77 (1971) 863-877.

[15] A. Katok, V. Nitica, A. Torok, Non-abelian cohomology of abelian Anosov actions, Ergodic Theory Dynamical Systems 20 (2000) 259-288.

[16] B. Lawson, Foliations, Bull. Amer. Math. Soc. 80 (1974) 369-418. 
[17] F. Ledrappier, Positivity of the exponent for stationary sequences of matrices, in : Lecture Notes in Math., Vol. 1186, 1986, pp. 56-73.

[18] F. Ledrappier, G. Royer, Croissance exponentielle de certains produits aléatoires de matrices, C. Acad. Sci. 280 (1980) 513-514.

[19] R. Leplaideur, Local product structure for equilibrium states, Trans. Amer. Math. Soc. 352 (2000) 1889-1912.

[20] V. Oseledets, A multiplicative ergodic theorem, Trans. Moscow Math. Soc. 19 (1968) 197231.

[21] W. Parry, M. Pollicott, Zeta functions and the periodic orbit structure of hyperbolic systems, Astérisque 187-188 (1990).

[22] V. Rokhlin, Sellected topics from the metric theory of dynamical systems, Amer. Math. Soc. Transl. 49 (1966) 171-240.

[23] G. Royer, Croissance exponentielle de produits markoviens de matrices aléatoires, Ann. Inst. Henri Poincaré 16 (1980) 49-62.

[24] D. Ruelle, A measure associated with Axiom A attractors, Amer. J. Math. 98 (1976) 619654.

[25] Ya. Sinai, Gibbs measures in ergodic theory, Russian Math. Surveys 27 (1972) 21-69. 